\title{
Exploration of Prognostic Biomarkers and Therapeutic Targets in The Microenvironment of Bladder Cancer Based on CXC Chemokines
}

\section{Xiaoqi Sun}

Department of Urology, Kaiping Central Hospital, Kaiping, 529300, China

\section{Qunxi Chen}

Department of Pathology, Sun Yat-sen University Cancer Center, Guangzhou, 510060, China Lihong Zhang

Department of Pathology, Sun Yat-sen University Cancer Center, Guangzhou, 510060, China Jiewei Chen

Department of Pathology, Sun Yat-sen University Cancer Center, Guangzhou, 510060, China

Xinke Zhang ( $\square$ zhangxk@sysucc.org.cn )

Sun Yat-sen University Cancer Center

\section{Research Article}

Keywords: Bladder cancer, Biomarkers, CXC Chemokines, Microenvironment

Posted Date: March 24th, 2021

DOI: https://doi.org/10.21203/rs.3.rs-223127/v4

License: (9) This work is licensed under a Creative Commons Attribution 4.0 International License. Read Full License 


\section{Abstract}

Bladder cancer (BLCA) has a high rate of morbidity and mortality, and is considered as one of the most malignant tumors of the urinary system. Tumor cells interact with surrounding interstitial cells, playing a key role in carcinogenesis and progression, which is partly mediated by chemokines. CXC chemokines exert anti-tumor biological roles in the tumor microenvironment and affect patient prognosis.

Nevertheless, their expression and prognostic values patients with BLCA remain unclear. We used online tools, including Oncomine, UALCAN, GEPIA, GEO databases, cBioPortal, GeneMANIA, DAVID 6.8, Metascape, TRUST (version 2.0), LinkedOmics, TCGA, and TIMER2.0 to perform the relevant analysis. The mRNA levels of C-X-C motif chemokine ligand (CXCL) 1, CXCL5, CXCL6, CXCL7, CXCL9, CXCL 10, CXCL11, CXCL13, CXCL16, and CXCL17 were increased significantly increased, and those of CXCL2, $C X C L 3$, and $C X C L 12$ were decreased significantly in BLCA tissues as assessed using the Oncomine, TCGA, and GEO databases. GEO showed that high levels of CXCL1, CXCL6, CXCL 10, CXCL 11, and CXCL13 mRNA expression are associated significantly with the poor overall survival (all $p<0.05$ ), and similarly, those of CXCL2 and CXCL 12 in the TCGA database $(p<0.05)$. The predominant signaling pathways involving the differentially expressed CXC chemokines are cell cycle, chemokine, and cytokinecytokine receptor interaction. Moreover, transcription factors such as Sp1 transcription factor (SP1), nuclear factor kappa B subunit 1 (NFKB1), and RELA proto-oncogene, NF-KB subunit (RELA) were likely play critical roles in regulating CXC chemokine expression. LYN proto-oncogene, src family tyrosine kinase (LYN) and LCK proto-oncogene, src family tyrosine kinase (LCK) were identified as the key targets of these CXC chemokines. MicroRNAs miR200 and miR30 were identified as the main microRNAs that interact with several CXC chemokines through an miRNA-target network. The expression of these chemokines is closely associated with the infiltration of six categories of immune cells. We explored the CXC chemokines superfamily-based biomarkers associated with BLCA prognosis using public databases, and provided possible chemokine targets for patients with BLCA.

\section{Introduction}

Bladder cancer (BLCA) is characterized by a high rate of morbidity and mortality, and is diagnosed in approximately 430,000 people per annum, of whom around 165,000 die of BLCA each year [1]. More than $90 \%$ of BLCA is considered to be urinary urothelial cancer according to its pathological histological subtype and smoking was identified as a risk factor for it formation [2]. Although the results of a prior clinical trial showed 5 - and 10 -year survival rates of approximately $50 \%$ and $36 \%$, respectively, for muscleinvasive BLCA using neoadjuvant chemotherapy [3], other studies showed 5-year survival rates ranging from $15 \%$ to $20 \%$ and high recurrence rates $[4,5]$. Therefore, for patients with high-risk non-metastatic BLCA, the recommended therapeutic strategy is radical cystectomy with lymph node dissection because of the poor efficacy of drug treatment, including maintenance bacille Calmette-Guérin (BCG) $[6,7]$. To date, tumor stage and lymph node $(\mathrm{LN})$ status have been identified as the most important prognostic factors after radical cystectomy [8], whereas in clinical practice, the value of other prognostic risk factors, including neutrophil-to-lymphocyte ratio, remains controversial $[9,10]$. Recently, molecular subtypes of 
BLCA derived from The Cancer Genome Atlas (TCGA) database attracted our attention. However, limited clinical value have restricted their application [11]. To date, although promising predictive biomarkers have been identified, they are not used routinely in clinical practice because of their finite values, and decision-making concerning therapy cannot be based only on these molecular markers, for example the tumor mutation burden, ribonucleic acid subtypes, and neutrophil-to-lymphocyte ratio [12]. Therefore, there is an urgent need to identify more therapeutic targets and prognostic biomarkers for use in patients with BLCA.

CXC chemokines represent the main component of approximately 50 chemokine family members, and their C-termini harbor a heparin-binding domain, which plays vital roles in the regulation of tumorassociated angiogenesis, tumor growth, and metastatic potential [13,14], in addition to mediating the migration of diversified leukocytes in non-tumor/tumor microenvironment (TME) [15-17]. The key role of CXC chemokines has been established as linking tumor development and metastasis; therefore, CXC chemokines and their receptors have been identified as therapeutic target in several cancers. Mounting experimental evidence shows that monoclonal antibodies against CXC chemokines or their receptors could inhibit tumor growth and/or metastasis, including breast cancer [18], non-Hodgkin lymphoma [19,] brain tumors [20], pancreatic tumors, and bladder tumors [21]. Therefore, CXC chemokines are likely to become therapeutic targets or prognostic factors in many tumors including BLCA.

Previous studies have revealed the functional role of several CXC chemokines in BLCA [22-26]. However, the identification of optimal prognostic biomarkers and therapeutic targets originating from $\mathrm{CXC}$ chemokines in BLCA remains an urgent and unresolved problem. Therefore, with the aim of fully comprehending the function of CXC chemokines in BLCA, the present study investigated the expression of CXC chemokines and their potential as prognostic biomarkers and therapeutic targets using exhaustive bioinformatic analyses based on several established databases. In addition, we provided evidence that will allow clinicians to choose appropriate therapeutic targets and accurately predict clinical outcome in patients with BLCA.

\section{Materials And Methods}

\section{Oncomine}

Oncomine (www.oncomine.org) integrates RNA and DNA-sequencing data from sources such as GEO, TCGA, and the published literature [27]. Screening of mRNA data allowed us to investigate CXC chemokine expression in BLCA. We set the significance thresholds of a p-value less than 1E-4, two-fold change, and overexpression or underexpression gene rank in top the $10 \%$. The differential expression of CXC chemokine mRNAs in BLCA was analyzed using Student's $t$ test.

UALCAN

UALCAN (http://ualcan.path.uab.edu/analysis.html) is an effective online analysis and mining website of tumor data, mainly based on the TCGA database, and can be used to perform biomarker identification, 
expression profile analysis, and survival analysis [28]. In this study, CXC chemokine mRNA expression data was obtained using the "Expression Analysis" module of the "BLCA" dataset. The differential expression of CXC chemokine mRNAs in BLCA was analyzed using Student's $t$ test.

GEPIA

GEPIA (http://gepia.cancer-pku.cn/index.html) is an analysis website of RNA sequencing data based on TCGA and GTEx databases, which was developed by Tang et al. [29]. We investigated the relationship between pathological stage and CXC chemokines with differential mRNA expression and their prognostic significance by screening Oncomine and UALCAN databases using the "Single Gene Analysis" module of GEPIA in BLCA.

\section{GEO databases}

GEO (Gene Expression Omnibus) databases collect and organize various expression chip data, and other chips, such as methylation chips, long noncoding RNA (IncRNA) chips, miRNA chips, chromosome number variation (CNV) chips, and even high-throughput sequencing data. CXC chemokine mRNA differential expression in BLCA was analyzed using Student's $t$ test and the corresponding prognostic analysis was performed using the statistical software GraphPad Prism 8.0.1 (GraphPad Inc., La Jolla, CA, USA).

cBioPortal

The data in cBioPortal (www.cbioportal.org) is derived from the International Cancer Genome Consortium (ICGC), TCGA, GEO, and other databases, which includes DNA methylation data, limited clinical data mRNA and microRNA expression data, non-synonymous mutations, protein level and phosphoprotein level (reverse-phase protein array (RPPA)) data, and DNA copy number data [30]. Based on TGCA data, cBioPortal provided genetic alterations and co-expression data for CXC chemokines. Bladder Urothelial Carcinoma (TCGA, Firehose Legacy) data from 413 cases were analyzed. Protein expression z scores (RPPA) and mRNA expression z scores (RNA Seq V2 (RNA-Seq by expectation-maximization (RSEM)) were entered using a $\mathrm{z}$ score threshold of \pm 2.0 .

GeneMANIA

The GeneMANIA plug-in of Cytoscape software is used to study protein-protein interactions [31].

STRING

STRING (https://string-db.org/) is an important software used to analyze protein-protein interactions [32]. To investigate the interactions of CXC chemokines, we constructed a network analysis of protein-protein interactions using STRING.

DAVID 6.8 
DAVID 6.8 (https://david.ncifcrf.gov/home.jsp) is used mainly for functional and pathway enrichment analysis for differentially expressed genes[33]. Differentially expressed CXC chemokines were analyzed using Kyoto Encyclopedia of Genes and Genomes (KEGG) pathway enrichment analysis and Gene Ontology (GO) enrichment analysis. The top 50 mutated genes of Bladder Urothelial Carcinoma (TCGA, Firehose Legacy; $p<0.05$ ) were analyzed using DAVID 6.8. The "ggplot2" package in the R project visualized the data.

Metascape

Metascape (http://metascape.org) is a powerful gene function annotation analysis tool, which can perform annotation enrichment for large quantities of genes or proteins, and construct protein-protein interaction networks [34]. Our study utilized the "Express Analysis" module to validate the enriched CXC chemokines and the top 50 mutated genes of Bladder Urothelial Carcinoma (TCGA, Firehose Legacy).

TRRUST (version 2)

The TRRUST (version 2) database (https://www.grnpedia.org/trrust/) records transcription factor regulatory relationships, including the target genes corresponding to transcription factors, and the regulatory relationships among transcription factors [35].

TIMER2.0

TIMER2.0 (http://timer.cistrome.org/) is a tumor immunity related database. "Gene module" refers to analyzing the association between CXC chemokine expression and immune cell types. "Clinical module" assesses the relationship among clinical outcome and tumor infiltrating immune cells and CXC chemokines [36].

\section{LinkedOmics}

LinkedOmics (http://www.linkedomics.org/) was used to analyze tumor multi-omics data for 32 TCGA tumor types [37]. "LinkInterpreter module" was used to obtain biological insights into transcription factor, miRNA, and kinase targets of enriched CXC chemokines. A minimum number of genes of 3 and a simulation of 500 were performed in Gene Set Enrichment Analysis (GSEA) for the BLCA dataset.

\section{Results}

\section{Chemokine expression status in patients with BLCA}

ONCOMINE database analysis allowed us to analyze the mRNA expression levels of 16 CXC chemokines (CXCL 1 to CXCL 14, CXCL16 and CXCL17) in BLCA and normal bladder mucosa tissues. Table 1 and Figure 1 show the results. ONCOMINE data demonstrated the mRNA expression levels of $C X C L 13$, CXCL10, CXCL9, and CXCL6 increased significantly and those of CXCL2, CXCL3, and CXCL12 decreased significantly in BLCA tissues compared with those in normal bladder mucosa tissues. This agreed with 
the results of Sanchez-Carbayo et al., who reported significantly increased mRNA levels of CXCL 13, CXCL 10, CXCL9, and CXCL6 in infiltrating bladder urothelial carcinoma and obviously decreased mRNA levels of $C X C L 2, C X C L 3$, and $C X C L 12$ in superficial bladder cancer [38]. Lee et al. observed lower CXCL 12 expression was in superficial bladder cancer (fold change $=-4.495$ and $p=2.52^{\mathrm{e}-21}$ ) and infiltrating bladder urothelial carcinoma (fold change $=-3.371$ and $p=1.41^{\mathrm{e}-12}$ ) compared with that in normal bladder mucosa [39].

Next, we used UALCAN to evaluate the mRNA expression levels of CXC chemokines in BLCA and normal bladder mucosa tissues, which showed markedly increased mRNA expression of $\operatorname{CXCL} 1\left(\mathrm{p}=8.50^{\mathrm{e}-03}\right)$, $\operatorname{CXCL5}\left(\mathrm{p}=1.12^{\mathrm{e}-06}\right), \operatorname{CXCL} 7\left(\mathrm{p}=1.42^{\mathrm{e}-02}\right), \operatorname{CXCL9}\left(\mathrm{p}=4.06^{\mathrm{e}-08}\right), \operatorname{CXCL} 10\left(\mathrm{p}=3.91^{\mathrm{e}-09}\right), \operatorname{CXCL} 11(\mathrm{p}=$ $\left.6.29^{\mathrm{e}-08}\right), C X C L 16\left(\mathrm{p}=1.44^{\mathrm{e}-03}\right)$, and $C X C L 17\left(\mathrm{p}=3.56^{\mathrm{e}-02}\right)$ in BLCA tissues, and significantly decreased mRNA levels of $C X C L 2\left(p=2.30^{\mathrm{e}-02}\right)$ and $C X C L 12\left(p=2.82^{\mathrm{e}-04}\right)$ (Figure 2). We also revealed that $C X C 17$ had the highest mRNA expression level in BLCA tissues based on the TCGA database (Figure 3). To investigate the role of the CXC chemokines in BLCA more comprehensively, including tumorigenesis and clinical prognosis, we assessed all the differentially expressed $C X C$ chemokines that were acquired by the Oncomine and TCGA databases including CXCL 1, CXCL2, CXCL3, CXCL5, CXCL6, CXCL7, CXCL9, CXCL10, CXCL11, CXCL12, CXCL13, CXCL16, and CXCL17.

\section{Prognostic Significance in patients with BLCA}

Next, we evaluated the relationship between the pathological stage of patients with BLCA and the differentially expressed CXC chemokines. We observed a significant association between $C X C L 2(\mathrm{p}=$ $0.0127)$ and $C X C L 12\left(p=8.68^{\mathrm{e}-07}\right)$ expression and pathological stage (Figure 4). CXCL2 and CXCL 12 mRNA expression levels were higher in more advanced pathological stages, indicating that they might associated with BLCA tumorigenesis. Meanwhile, GEPIA was used to assess the impact of differentially expressed CXC chemokines on clinical outcome. Patients with BLCA with high CXCL2 ( $p=0.17), C X C L 16$ $(p=0.14)$, and $C X C L 13(p=0.12)$ mRNA expression had a statistical tendency towards longer diseasefree survival (Figure 5). In addition, high mRNA levels of $\operatorname{CXCL} 1(p=0.17)$ and $\operatorname{CXCL} 12(p=0.0075)$ had a statistical tendency towards shorter overall survival (Figure 5).

GEO database analysis demonstrated that CXCL3 mRNA expression $(p=0.034)$ was higher in BLCA tissues than in normal tissues. $C X C L 12(p<0.001), C X C L 9(p=0.002)$, and $C X C L 10(p=0.015)$ mRNA expression levels were higher in normal tissues than in BLCA tissues (Figure 11). Survival analysis showed that high levels of $C X C L 1, C X C L 6, C X C L 10, C X C L 11$, and $C X C L 13$ mRNA expression were associated significantly with the poor overall survival (all $p<0.05$ ). High levels of $C X C L 3$ mRNA had the statistic tendency towards predicting shorter overall survival $(p=0.053)$ (Figure 12).

CXC Chemokine Interaction Analyses, Neighbor Gene Network, Co-expression, and Genetic Alteration in patients with BLCA 
We analyzed comprehensively the molecular characteristics of the differentially expressed CXC chemokines. First, we used TCGA datasets to analyze their genetic alterations, which showed the rates of genetic alteration of CXCL 1, CXCL2, CXCL3, CXCL5, CXCL6, CXCL7, CXCL9, CXCL 10, CXCL 11, CXCL12, CXCL13, CXCL16, and CXCL17 were 6, 5, 4, 5, 5, 4, 5, 6, 5, 2.7, 3, 5, and 11\%, respectively in the BLCA samples queried (Figure 7A). In the BLCA samples, the most common alterations were amplification and high mRNA expression. Differentially expressed CXC chemokine co-expression analysis identified strong correlations among $C X C L 9, C X C L 10$, and $C X C L 11$ expression; moderate to strong correlations among CXCL1, CXCL2, CXCL3, CXCL5, and CXCL6; and weak to moderate correlations among CXCL7, CXCL 12, CXCL13, CXCL16, and CXCL 17 expression (Figure 7B). Secondly, PPI network analysis with STRING obtained 13 nodes and 71 edges in the analysis of the interactions among the differentially expressed CXC chemokines (Figure 7D). Enrichment pathways identified in STRING for these CXC chemokines included the TNF signaling pathway, the IL-17 signaling pathway, cytokine-cytokine receptor interactions, and the chemokine signaling pathway. The results of analysis at GeneMANIA revealed that their functions were related to chemokine receptor binding, cell chemotaxis, and chemokine activity (Figure 7E).

In addition, the top 50 mutated genes of 200 most mutated cases with BLCA were identified using cBioPortal (Figure 7C), representing mutations in which lead to the formation of a tumor-associated antigen that is associated with the TME and is closely associated with the CXC chemokines. Next, we analyzed the associations of these mutated genes and the CXC chemokines. These top 50 mutated genes have close interactions with the differentially expressed CXC chemokines in BLCA (Figure 7F).

\section{Functional Enrichment Analysis of CXC Chemokines in Patients with BLCA}

The functions of differentially expressed CXC chemokines and the top 50 mutated genes in BLCA were analyzed by DAVID6.8 and Metascape. The top $10 \mathrm{GO}$ enriched terms in the biological process (BP), cellular component (CC), and molecular function (MF) categories were analyzed using DAVID 6.8. Chemokine-mediated signaling pathway, positive regulation of leukocyte chemotaxis, response to lipopolysaccharide, regulation of cell proliferation, inflammatory response, immune response, positive regulation of CAMP metabolic process, and cell-cell signaling were related to the development and progression of BLCA (Figure 8A). The top $10 \mathrm{GO}$ terms in the CC category included, $Z$ disc, sarcolemma, extracellular space, sarcoplasmic reticulum membrane, sarcoplasmic reticulum, extracellular region, $\mathrm{T}$ tubule, MLL3/4 complex, costamere, and histone methyltransferase complex (Figure 8B). Chemokine activity and CXCR chemokine receptor binding were the two most significantly enriched GO terms in the MF category (Figure $8 \mathrm{C}$ ). The top $10 \mathrm{KEGG}$ pathways were the chemokine signaling pathway, cytokinecytokine receptor interaction, cell cycle, the TNF signaling pathway, and microRNAs in cancer, HIF-1 signaling pathway, pathways in cancer, viral carcinogenesis, central carbon metabolism in cancer, which were intimately associated with BLCA tumorigenesis (Figure 8D).

Metascape analysis showed that the functions of the differentially expressed CXC chemokines and top 50 mutated genes in BLCA were predominantly enriched in chemokine receptor bind chemokines, $\mathrm{T}$ cell 
chemotaxis, and microRNAs in cancer (Figure 9A, B). Next, we extracted protein-protein interaction (PPI) and MCODE components to explore the correlations between BLCA and CXC chemokines in detail; the list of identified genes is shown in Figure 9C, D. Three most significant MCODE components were obtained and showed that the biological functions were involved in chemokine receptor binding to chemokines, chemokine-mediated signaling pathways, chemokine signaling pathways, activation of homeobox (HOX) genes during differentiation, and histone modification (Figure 9E).

\section{MiRNA Targets, Kinase Targets, and Transcription Factor Targets of CXC chemokines in patients with BLCA}

Next, we investigated the transcription factor and kinase targets of the differentially expressed chemokines by analyses in the TRRUST and LinkedOmics databases. TRRUST analysis of $C X C L 1$, CXCL2, CXCL5, CXCL7, CXCL10, and CXCL12 identified three transcription factors: Sp1 transcription factor (SP1), nuclear factor kappa B subunit 1 (NFKB1), and RELA proto-oncogene, NF-KB subunit (RELA) that were possibly related CXC chemokine regulation (Table 2). Critically, NFKB1 and RELA mediated the expression of CXCL1, CXCL2, CXCL5, CXCL10, and CXCL12, and SP1 mediated the expression of CXCL1 and CXCL5. Next, LinkedOmics database analysis was used to identify the top two kinase targets of these CXC chemokines (Table 3). LYN proto-oncogene, src family tyrosine kinase (LYN) was identified as the kinase target of CXCL1, CXCL2, CXCL3, CXCL5, CXCL6, CXCL9, CXCL10, CXCL11, CXCL12, and CXCL 13 in the kinase-target network, and LCK proto-oncogene, src family tyrosine kinase (LCK) was identified as the kinase target of CXCL2, CXCL3, CXCL5, CXCL6,CXCL9, CXCL10, CXCL11, CXCL 13, and CXCL16. Inhibitor of nuclear factor kappa $B$ kinase subunit beta (IKBKB), protein kinase CGMP-dependent 1 (PRKG1), and Janus kinase 2 (JAK2) were identified as key kinase targets of CXCL 1, CXCL12, and CXCL16, respectively, in the kinase-target network. Moreover, protein kinase N1 (PKN1), adrenergic, beta, receptor kinase 1 (ADRBK1) and checkpoint kinase 1 (CHEK1), polo like kinase 1 (PLK1) were identified as the top two target kinase for $C X C L 7$ and $C X C L 17$, respectively. Finally, analysis at the LinkedOmics database allowed us to identify miRNAs that targeted the mRNAs encoding CXC chemokines (Table 4). The miRNA miR200A interacted mainly with the CXCL 1 and CXCL5 miRNA-target network. Chemokine mRNAs CXCL 1 and CXCL6 might be targeted by miR-30E-5P, miR-30B, miR-30D, miR-30C, and miR-30A5P. CXCL3 expression might be modulated by miR-409-3P and miR-526B. CXCL5 and CXCL6 might be targeted by miR-141 and miR-374, respectively. CXCL7 might be targeted by miR-154, miR-487, miR-525, and miR-524s. Finally, CXCL 17 might be targeted by miR-490.

\section{Immune Cell Infiltration of CXC Chemokines in patients with BLCA Patients}

CXC chemokines influence immune cell infiltration and inflammatory responses in the TME, thus possibly affecting the clinical therapy and outcome in patients with BLCA. Using the TIMER database, we investigated comprehensively the correlation between infiltration of various immune cells and the differentially expressed CXC chemokines. CXCL 1 expression correlated positively with the infiltration of dendritic cells (Cor $=0.427, p=1.43^{e^{-17}}$, neutrophils (Cor $\left.=0.444, p=5.46^{e-19}\right), C D 4+T$ cells $($ Cor $=0.112$, $\left.p=3.26^{e-02}\right)$, and CD8+ T cells (Cor $\left.=0.303, p=3.39^{e^{-09}}\right)$; Figure $\left.9 A\right)$. Similarly, CXCL2 expression 
correlated positively with the infiltration of dendritic cells (Cor $=0.345, p=1.22^{\mathrm{e}-11}$, neutrophils (Cor = $0.355, p=3.05^{e-12}$ ), CD4+ T cells (Cor $=0.128, p=1.46^{\mathrm{e}-02}$ ), and CD8+ T cells (Cor $=0.255, p=7.61^{\mathrm{e}-07}$; Figure 9B). CXCL3 expression correlated negatively with the infiltration of dendritic cells (Cor $=0.4, p=$ $1.93^{\mathrm{e}-15}$, neutrophils (Cor $=0.424, p=2.86^{\mathrm{e}-17}$ ), CD4+ T cells (Cor $=0.108, p=3.89^{\mathrm{e}-02}$ ), CD8+ T cells (Cor $=0.302, p=3.55^{\mathrm{e}-09}$ ), and B cells (Cor $\left.=-0.103, p=4.88^{\mathrm{e}-02}\right)$; Figure $9 C$ ). CXCL 5 expression correlated positively with the infiltration of dendritic cells $\left(\mathrm{Cor}=0.43, p=7.83^{\mathrm{e}-18}\right)$, neutrophils $(\mathrm{Cor}=0.385, \mathrm{p}=$ $\left.2.70^{\mathrm{e}-14}\right), \mathrm{CD} 4+\mathrm{T}$ cells (Cor $\left.=0.116, \mathrm{p}=2.63^{\mathrm{e}-02}\right), \mathrm{CD} 8+\mathrm{T}$ cells $\left(\right.$ Cor $=0.288, \mathrm{p}=2.10^{\mathrm{e}-08}$; Figure 9D). CXCL6 expression correlated positively with the infiltration of CD8+ T cells $\left(\right.$ Cor $\left.=0.219, p=2.42^{e^{-05}}\right)$, neutrophils (Cor $=0.339, p=3.50^{\mathrm{e}^{-11}}$ ), and dendritic cells (Cor $=0.43, p=7.83^{\mathrm{e}-18}$; Figure 9E). A similar

correlation tendency was found between CXCL7 expression and dendritic cells (Cor $=0.239, p=3.74^{\mathrm{e}-06}$ ), neutrophils (Cor $=0.174, p=8.87^{\mathrm{e}-04}$ ), and CD8+ T cells (Cor $=0.164, p=1.60^{\mathrm{e}-03}$; Figure 9F). CXCL9, CXCL10, and CXCL11 mRNA expression levels correlated positively with the infiltration of dendritic cells, neutrophils, CD4+ T cells, and CD8+ T cells (all $p<0.05$; Figure 9G-I). CXCL 12 expression correlated positively with the infiltration of dendritic cells (Cor $\left.=0.156, p=2.88^{\mathrm{e}-03}\right)$, neutrophils $($ Cor $=0.208, p=$ $6.27^{\mathrm{e}-05}$ ), macrophages (Cor $\left.=0.464, \mathrm{p}=7.25^{\mathrm{e}-21}\right), \mathrm{CD} 4+\mathrm{T}$ cells (Cor $=0.146, \mathrm{p}=5.21^{\mathrm{e}-03}$ ), and CD8+ $\mathrm{T}$ cells (Cor $=0.134, p=1.05^{\mathrm{e}-02}$; Figure $9 \mathrm{~J}$ ). With the exception of macrophages, CXCL 13 expression correlated positively associated with the other immune cell types consisted of dendritic cells, CD4+ $T$ cells, neutrophils, CD8+ T cells, and B cells ( $p<0.05$; Figure 9K). CXCL 16 expression correlated positively with the infiltration of CD8+ T cells, CD4+ T cells, macrophages, neutrophils, and dendritic cells (all $p<$ 0.05; Figure 9L). CXCL 17 mRNA expression correlated positively with B cells (Cor $=0.156, p=2.83^{\mathrm{e}-03}$ ), and negatively with dendritic cells (Cor $=-0.167, p=1.35^{\mathrm{e}-03}$; Figure $9 \mathrm{M}$ ). A Cox proportional hazard model was used identify factors associated with prognosis of patients with BLCA, which identified $B$ cells $(p=0.032)$, macrophages $(p=0.001)$, and $C X C L 2$ mRNA expression $(p=0.010) . C X C L 7$ mRNA expression $(p=0.066)$ had significantly statistical tendency towards predicting the clinical outcome of BLCA patients (Table 5).

\section{Discussion}

Preclinical data indicate that bladder cancer is one of the most immunogenic tumors [40], which is assumed to make it sensitive to favorable targeted therapies, not including PD-1/PDL-1 checkpoint inhibitors. Previous studies have unveiled a TME-related role of CXC chemokines [41], and functions in the immunotherapy of cancer [42]. Chemokines are involved in the process of cancer biology, including tumor proliferation and metastasis, and targeting of chemokines and chemokine receptors have been evaluated in several preclinical studies and clinical trials, such as in breast cancer [43], pancreatic cancer [44], renal tumor [45], and prostate cancer [46]. Nonetheless, in the prognosis and biology of BLCA, the significance of CXC chemokines are poorly understood.

The present study characterized the mRNA expression patterns CXC chemokines and investigated their pathological relationship with BLCA stages. Oncomine and TCGA database analysis identified 13 CXC 
chemokines that were differentially expressed in BLCA tissues compared with that in normal tissues, including upregulated expression (CXCL 1, CXCL5, CXCL6, CXCL7, CXCL9, CXCL 10, CXCL 11, CXCL 13, CXCL16, and CXCL17) and downregulated expression (CXCL2, CXCL3, and CXCL12). In addition, the TCGA analysis results showed that high expression of CXCL2 and CXCL12 was associated with advanced pathological stage, and analysis of the GEO and TCGA databases revealed that high levels of CXCL1, CXCL6, CXCL10, CXCL11, CXCL12, and CXCL13 mRNA expression were associated significantly with poor overall survival, indicating these differentially expressed $C X C$ chemokines exert important roles in the proliferation and development of BLCA. This agreed partially with the results of previous studies, which reported that $C X C L 5$ promotes chemoresistance in BLCA cells [47] and CXCL 12 is associated with the genesis and development of BLCA [23].

We next investigated the differentially expressed CXC chemokines for their molecular characteristics in BLCA. Their most frequent genetic alterations were high mRNA expression and amplification, following by deep deletion, missense mutation, and truncating mutation, suggesting that genetic alterations might play a vital role in tumorigenesis and progression of BLCA. In particular, high mRNA expression and amplification of CXC chemokine genes could have the predominant impact on the BLCA processes. Previous studies reported that overexpression of several chemokines, including CXCL1, CXCL2, CXCL3, CXCL4, CXCL5, CXCL6, CXCL7, and CXCL8, was involved in breast cancer and melanoma metastasis, resulting from enhanced gene transcription [48] and copy number amplification of their encoding genes [49]. Moreover, mutants of CXCL8 had lower potency and efficacy in chemotaxis assays using neutrophils [50]. Together, our results and those of previous studies suggest the critical role of CXC chemokines in the tumorigenesis and cancer progression.

We next used GO and KEGG analysis to provide clues to the biological functions of these CXC chemokines. The results showed the CXC chemokines and top 50 mutated genes in BLCA are functionally associated with the TNF pathway, cell cycle, cytokine-cytokine receptor interactions, and the chemokine signaling pathway. Tumor metastasis, immune evasion, angiogenesis, and proliferation are reported to be associated critically with chemokine signaling pathways [51, 52]. The TNF signaling pathway responds to cellular stress and inflammatory signals to activate cytokine cascades, which further affect the immune microenvironment. In addition, TNF interacts with different receptors to mediate cellular apoptosis or growth $[53,54]$. Moreover, the TNF signaling pathway contributes to tumor growth and metastasis in various cancers and acts as a therapeutic target [55-58]. These pieces of evidence showed that CXC chemokines might be developed as effective drug targets to treat patients with BLCA in the future.

We explored the transcription factor targets of the differentially expressed CXC chemokines, their kinase targets, and miRNAs that targeted them. We observed that SP1, NFKB1, and RELA might regulate the expression of CXC chemokines. RELA regulates breast cancer progression via the CXCL 13/CXCR5 axis [59]. In addition, via the IRF1-CDK4 signaling axis, RELA-induced Interferon- $y$ inhibits the proliferation of breast cancer [60]. RELA also regulates oncogene-induced senescence to decrease the proliferation in murine Kras-driven pancreatic cancer via the CXCL1/CXCR2 axis [61]. Additionally, RELA acts as an important transcription factor in the activation and stability of regulatory $T$ cells [62] and regulates Th17 
differentiation by miR-30a in experimental autoimmune encephalomyelitis [63]. NFKB1 is involved widely in carcinogenesis, including driving tumor progression or acting as a tumor-suppressor by phosphorylation and ubiquitination pathways [64]. Polymorphisms of the NFKB1 promoter are associated with a higher risk of bladder cancer [65], and aberrant NFKB1 activity leads to impaired M1 polarization of macrophages by repressing the expression of CXCL 10 and $I L-12$, which forms an immunosuppressive microenvironment to induce the immune escape of tumors [64]. Our study provided evidence that there is an association between CXC chemokines and the NF-KB signaling pathway during BLCA tumorigenesis and development. Our results also suggested Src family tyrosine kinases (LCK and LYN), PKN1, PRKG1, and CHEK1 might be the targets of the differential CXC chemokines, which play important roles in cell growth, division, migration, and survival signaling pathways [66-69], and mediate the carcinogenesis of several tumors [70-72]. Therefore, these differentially expressed CXC chemokines might modulate BLCA development and progression dependent on the above-mentioned signaling pathways by regulating these kinases.

We found the miR-30 family is the main miRNA family targeting CXC chemokines such as CXCL 1 and CXCL6 in BLCA, suggesting that this miRNA family plays crucial regulatory roles in BLCA carcinogenesis and progression by acting on mRNAs encoding CXC chemokines. This hypothesis was supported partially by previous reports that the miR-30 family is associated with the development of organs and the pathogenesis of diseases, including various cancers, such as breast cancer and colorectal cancer [73-75].

Chemokines function by mediating the migration of immune cells by interacting with their cognate ligands [41]. Immune cell infiltration, especially CD8+ T cells, could affect tumor progression, as well as predicting the sensitivity to immunotherapy and clinical prognosis $[76,77]$. The present study indicated that there is a distinct correlation between infiltrating immune cells (e.g., dendritic cells, neutrophils, macrophages, CD4+ T cells, CD8+ T cells, and B cells) and the differentially expressed CXC chemokines, suggesting that these chemokines act as crucial components reflecting the immune status in the TME and could provide evidence of drug targeting in BLCA immunotherapy in the future.

\section{Conclusion}

We analyzed the relationship between the CXC chemokines and the 50 top mutated genes in patients with BLCA and explored the CXC chemokines superfamily-based biomarkers associated with BLCA prognosis by public databases. The results of these analyses identified possible target chemokines that could lead to novel drug discovery or immunotherapy to treat patients with BLCA in the future.

\section{Declarations}

\section{Acknowledgements}

We appreciate the generosity of TCGA and GEO databases for sharing the mounting clinical and genetic information. This study was supported by the Medical Science Funding of Guangdong province 
(A2020139). We have updated version of the manuscript.

\section{Data Availability}

All the data sets used in this study were publicly available at https://xenabrowser.net and http://www.ncbi.nlm.nih.gov/geo.

\section{Conflicts of Interest}

The authors declare no competing financial interests.

\section{Author Contributions}

Xinke Zhang and Jiewei Chen designed this study. Xiaoqi Sun and Qunxi Chen analyzed the data and wrote the manuscript. Lihong Zhang analyzed the data. Xinke Zhang revised the manuscript. All authors approved the final version for submission.

\section{References}

1. Antoni S, Ferlay J, Soerjomataram I, Znaor A, Jemal A, Bray F: Bladder Cancer Incidence and Mortality: A Global Overview and Recent Trends. Eur Urol 2017, 71(1):96-108.

2. Ferlay J, Soerjomataram I, Dikshit R, Eser S, Mathers C, Rebelo M, Parkin DM, Forman D, Bray F: Cancer incidence and mortality worldwide: sources, methods and major patterns in GLOBOCAN 2012. Int J Cancer 2015, 136(5):E359-386.

3. International Collaboration of T, Medical Research Council Advanced Bladder Cancer Working P, European Organisation for R, Treatment of Cancer Genito-Urinary Tract Cancer G, Australian Bladder Cancer Study G, National Cancer Institute of Canada Clinical Trials G, Finnbladder, Norwegian Bladder Cancer Study G, Club Urologico Espanol de Tratamiento Oncologico G, Griffiths G et al: International phase III trial assessing neoadjuvant cisplatin, methotrexate, and vinblastine chemotherapy for muscle-invasive bladder cancer: long-term results of the BA06 30894 trial. J Clin Oncol 2011, 29(16):2171-2177.

4. Bladder cancer: diagnosis and management of bladder cancer: (c) NICE (2015) Bladder cancer: diagnosis and management of bladder cancer. BJU Int 2017, 120(6):755-765.

5. Aggen DH, Drake CG: Biomarkers for immunotherapy in bladder cancer: a moving target. $J$ Immunother Cancer 2017, 5(1):94.

6. Babjuk M, Burger M, Comperat EM, Gontero P, Mostafid AH, Palou J, van Rhijn BWG, Roupret M, Shariat SF, Sylvester R et al: European Association of Urology Guidelines on Non-muscle-invasive Bladder Cancer (TaT1 and Carcinoma In Situ) - 2019 Update. Eur Urol 2019, 76(5):639-657.

7. Cambier S, Sylvester RJ, Collette L, Gontero P, Brausi MA, van Andel G, Kirkels WJ, Silva FC, Oosterlinck W, Prescott S et al: EORTC Nomograms and Risk Groups for Predicting Recurrence, Progression, and Disease-specific and Overall Survival in Non-Muscle-invasive Stage Ta-T1 Urothelial 
Bladder Cancer Patients Treated with 1-3 Years of Maintenance Bacillus Calmette-Guerin. Eur Urol 2016, 69(1):60-69.

8. Dutta R, Abdelhalim A, Martin JW, Vernez SL, Faltas B, Lotan Y, Youssef RF: Effect of tumor location on survival in urinary bladder adenocarcinoma: A population-based analysis. Urol Oncol 2016, 34(12):531 e531-531 e536.

9. Wu S, Zhao X, Wang Y, Zhong Z, Zhang L, Cao J, Ai K, Xu R: Pretreatment Neutrophil-Lymphocyte Ratio as a Predictor in Bladder Cancer and Metastatic or Unresectable Urothelial Carcinoma Patients: a Pooled Analysis of Comparative Studies. Cell Physiol Biochem 2018, 46(4):1352-1364.

10. Ojerholm E, Smith A, Hwang WT, Baumann BC, Tucker KN, Lerner SP, Mamtani R, Boursi B, Christodouleas JP: Neutrophil-to-lymphocyte ratio as a bladder cancer biomarker: Assessing prognostic and predictive value in SWOG 8710. Cancer 2017, 123(5):794-801.

11. Kamoun A, de Reynies A, Allory Y, Sjodahl G, Robertson AG, Seiler R, Hoadley KA, Groeneveld CS, AlAhmadie H, Choi W et al: A Consensus Molecular Classification of Muscle-invasive Bladder Cancer. Eur Urol 2020, 77(4):420-433.

12. Witjes JA, Bruins HM, Cathomas R, Comperat EM, Cowan NC, Gakis G, Hernandez V, Linares Espinos E, Lorch A, Neuzillet $Y$ et al: European Association of Urology Guidelines on Muscle-invasive and Metastatic Bladder Cancer: Summary of the 2020 Guidelines. Eur Urol 2020.

13. Gerber PA, Hippe A, Buhren BA, Muller A, Homey B: Chemokines in tumor-associated angiogenesis. Biol Chem 2009, 390(12):1213-1223.

14. Keeley EC, Mehrad B, Strieter RM: CXC chemokines in cancer angiogenesis and metastases. Adv Cancer Res 2010, 106:91-111.

15. Luster AD: Chemokines-chemotactic cytokines that mediate inflammation. N Engl J Med 1998, 338(7):436-445.

16. Struyf S, Proost P, Van Damme J: Regulation of the immune response by the interaction of chemokines and proteases. Adv Immunol 2003, 81:1-44.

17. Keeley EC, Mehrad B, Strieter RM: Chemokines as mediators of neovascularization. Arterioscler Thromb Vasc Biol 2008, 28(11):1928-1936.

18. Muller A, Homey B, Soto H, Ge N, Catron D, Buchanan ME, McClanahan T, Murphy E, Yuan W, Wagner $\mathrm{SN}$ et al: Involvement of chemokine receptors in breast cancer metastasis. Nature 2001, 410(6824):50-56.

19. Bertolini F, Dell'Agnola C, Mancuso P, Rabascio C, Burlini A, Monestiroli S, Gobbi A, Pruneri G, Martinelli G: CXCR4 neutralization, a novel therapeutic approach for non-Hodgkin's lymphoma. Cancer Res 2002, 62(11):3106-3112.

20. Rubin JB, Kung AL, Klein RS, Chan JA, Sun Y, Schmidt K, Kieran MW, Luster AD, Segal RA: A smallmolecule antagonist of CXCR4 inhibits intracranial growth of primary brain tumors. Proc Natl Acad Sci U S A 2003, 100(23):13513-13518.

21. Vandercappellen J, Van Damme J, Struyf S: The role of CXC chemokines and their receptors in cancer. Cancer Lett 2008, 267(2):226-244. 
22. Miyake M, Hori S, Morizawa Y, Tatsumi Y, Nakai Y, Anai S, Torimoto K, Aoki K, Tanaka N, Shimada K et al: CXCL1-Mediated Interaction of Cancer Cells with Tumor-Associated Macrophages and CancerAssociated Fibroblasts Promotes Tumor Progression in Human Bladder Cancer. Neoplasia 2016, 18(10):636-646.

23. Nazari A, Khorramdelazad H, Hassanshahi G: Biological/pathological functions of the CXCL12/CXCR4/CXCR7 axes in the pathogenesis of bladder cancer. Int J Clin Onco/2017, 22(6):9911000.

24. Zhang H, Ye YL, Li MX, Ye SB, Huang WR, Cai TT, He J, Peng JY, Duan TH, Cui J et al: CXCL2/MIFCXCR2 signaling promotes the recruitment of myeloid-derived suppressor cells and is correlated with prognosis in bladder cancer. Oncogene 2017, 36(15):2095-2104.

25. Nazari A, Ahmadi Z, Hassanshahi G, Abbasifard M, Taghipour Z, Falahati-Pour SK, Khorramdelazad H: Effective Treatments for Bladder Cancer Affecting CXCL9/CXCL10/CXCL11/CXCR3 Axis: A Review. Oman Med J 2020, 35(2):e103.

26. Zheng J, Zhu X, Zhang J: CXCL5 knockdown expression inhibits human bladder cancer T24 cells proliferation and migration. Biochem Biophys Res Commun 2014, 446(1):18-24.

27. Rhodes DR, Yu J, Shanker K, Deshpande N, Varambally R, Ghosh D, Barrette T, Pandey A, Chinnaiyan AM: ONCOMINE: a cancer microarray database and integrated data-mining platform. Neoplasia 2004, 6(1):1-6.

28. Chandrashekar DS, Bashel B, Balasubramanya SAH, Creighton CJ, Ponce-Rodriguez I, Chakravarthi B, Varambally S: UALCAN: A Portal for Facilitating Tumor Subgroup Gene Expression and Survival Analyses. Neoplasia 2017, 19(8):649-658.

29. Tang Z, Li C, Kang B, Gao G, Li C, Zhang Z: GEPIA: a web server for cancer and normal gene expression profiling and interactive analyses. Nucleic Acids Res 2017, 45(W1):W98-W102.

30. Gao J, Aksoy BA, Dogrusoz U, Dresdner G, Gross B, Sumer SO, Sun Y, Jacobsen A, Sinha R, Larsson E et al: Integrative analysis of complex cancer genomics and clinical profiles using the cBioPortal. Sci Signal 2013, 6(269):pl1.

31. Warde-Farley D, Donaldson SL, Comes O, Zuberi K, Badrawi R, Chao P, Franz M, Grouios C, Kazi F, Lopes CT et al: The GeneMANIA prediction server: biological network integration for gene prioritization and predicting gene function. Nucleic Acids Res 2010, 38(Web Server issue):W214-220.

32. Szklarczyk D, Gable AL, Lyon D, Junge A, Wyder S, Huerta-Cepas J, Simonovic M, Doncheva NT, Morris JH, Bork P et al: STRING v11: protein-protein association networks with increased coverage, supporting functional discovery in genome-wide experimental datasets. Nucleic Acids Res 2019, 47(D1):D607-D613.

33. Huang da W, Sherman BT, Lempicki RA: Systematic and integrative analysis of large gene lists using DAVID bioinformatics resources. Nat Protoc 2009, 4(1):44-57.

34. Zhou Y, Zhou B, Pache L, Chang M, Khodabakhshi AH, Tanaseichuk O, Benner C, Chanda SK: Metascape provides a biologist-oriented resource for the analysis of systems-level datasets. Nat Commun 2019, 10(1):1523. 
35. Han H, Cho JW, Lee S, Yun A, Kim H, Bae D, Yang S, Kim CY, Lee M, Kim E et al: TRRUST v2: an expanded reference database of human and mouse transcriptional regulatory interactions. Nucleic Acids Res 2018, 46(D1):D380-D386.

36. Li T, Fu J, Zeng Z, Cohen D, Li J, Chen Q, Li B, Liu XS: TIMER2.0 for analysis of tumor-infiltrating immune cells. Nucleic Acids Res 2020, 48(W1):W509-W514.

37. Vasaikar SV, Straub P, Wang J, Zhang B: LinkedOmics: analyzing multi-omics data within and across 32 cancer types. Nucleic Acids Res 2018, 46(D1):D956-D963.

38. Sanchez-Carbayo M, Socci ND, Lozano J, Saint F, Cordon-Cardo C: Defining molecular profiles of poor outcome in patients with invasive bladder cancer using oligonucleotide microarrays. J Clin Oncol 2006, 24(5):778-789.

39. Lee JS, Leem SH, Lee SY, Kim SC, Park ES, Kim SB, Kim SK, Kim YJ, Kim WJ, Chu IS: Expression signature of E2F1 and its associated genes predict superficial to invasive progression of bladder tumors. J Clin Oncol 2010, 28(16):2660-2667.

40. Lawrence MS, Stojanov P, Polak P, Kryukov GV, Cibulskis K, Sivachenko A, Carter SL, Stewart C, Mermel $\mathrm{CH}$, Roberts SA et al: Mutational heterogeneity in cancer and the search for new cancerassociated genes. Nature 2013, 499(7457):214-218.

41. Susek KH, Karvouni M, Alici E, Lundqvist A: The Role of CXC Chemokine Receptors 1-4 on Immune Cells in the Tumor Microenvironment. Front Immunol 2018, 9:2159.

42. Mollica Poeta V, Massara M, Capucetti A, Bonecchi R: Chemokines and Chemokine Receptors: New Targets for Cancer Immunotherapy. Front Immunol 2019, 10:379.

43. Schott AF, Goldstein LJ, Cristofanilli M, Ruffini PA, McCanna S, Reuben JM, Perez RP, Kato G, Wicha M: Phase lb Pilot Study to Evaluate Reparixin in Combination with Weekly Paclitaxel in Patients with HER-2-Negative Metastatic Breast Cancer. Clin Cancer Res 2017, 23(18):5358-5365.

44. Nywening TM, Wang-Gillam A, Sanford DE, Belt BA, Panni RZ, Cusworth BM, Toriola AT, Nieman RK, Worley LA, Yano $M$ et al: Targeting tumour-associated macrophages with CCR2 inhibition in combination with FOLFIRINOX in patients with borderline resectable and locally advanced pancreatic cancer: a single-centre, open-label, dose-finding, non-randomised, phase 1b trial. Lancet Oncol 2016, 17(5):651-662.

45. Berlato C, Khan MN, Schioppa T, Thompson R, Maniati E, Montfort A, Jangani M, Canosa M, Kulbe H, Hagemann UB et al: A CCR4 antagonist reverses the tumor-promoting microenvironment of renal cancer. J Clin Invest 2017, 127(3):801-813.

46. Di Mitri D, Toso A, Chen JJ, Sarti M, Pinton S, Jost TR, D'Antuono R, Montani E, Garcia-Escudero R, Guccini I et al: Tumour-infiltrating Gr-1+ myeloid cells antagonize senescence in cancer. Nature 2014, 515(7525):134-137.

47. Wang C, Li A, Yang S, Qiao R, Zhu X, Zhang J: CXCL5 promotes mitomycin C resistance in nonmuscle invasive bladder cancer by activating EMT and NF-kappaB pathway. Biochem Biophys Res Commun 2018, 498(4):862-868. 
48. Bieche I, Chavey C, Andrieu C, Busson M, Vacher S, Le Corre L, Guinebretiere JM, Burlinchon S, Lidereau R, Lazennec G: CXC chemokines located in the 4q21 region are up-regulated in breast cancer. Endocr Relat Cancer 2007, 14(4):1039-1052.

49. Yang XR, Brown K, Landi MT, Ghiorzo P, Badenas C, Xu M, Hayward NK, Calista D, Landi G, Bruno W et al: Duplication of CXC chemokine genes on chromosome 4q13 in a melanoma-prone family. Pigment Cell Melanoma Res 2012, 25(2):243-247.

50. Lusti-Narasimhan M, Power CA, Allet B, Alouani S, Bacon KB, Mermod JJ, Proudfoot AE, Wells TN: Mutation of Leu25 and Val27 introduces CC chemokine activity into interleukin-8. J Biol Chem 1995, 270(6):2716-2721.

51. Lim SY, Yuzhalin AE, Gordon-Weeks AN, Muschel RJ: Targeting the CCL2-CCR2 signaling axis in cancer metastasis. Oncotarget 2016, 7(19):28697-28710.

52. Sarvaiya PJ, Guo D, Ulasov I, Gabikian P, Lesniak MS: Chemokines in tumor progression and metastasis. Oncotarget 2013, 4(12):2171-2185.

53. Kischkel FC, Lawrence DA, Tinel A, LeBlanc H, Virmani A, Schow P, Gazdar A, Blenis J, Arnott D, Ashkenazi A: Death receptor recruitment of endogenous caspase-10 and apoptosis initiation in the absence of caspase-8. J Biol Chem 2001, 276(49):46639-46646.

54. Chen X, Subleski JJ, Kopf H, Howard OM, Mannel DN, Oppenheim JJ: Cutting edge: expression of TNFR2 defines a maximally suppressive subset of mouse CD4+CD25+FoxP3+ T regulatory cells: applicability to tumor-infiltrating T regulatory cells. J Immuno/ 2008, 180(10):6467-6471.

55. Tanaka T, Imamura T, Yoneda M, Irie A, Ogi H, Nagata M, Yoshida R, Fukuma D, Kawahara K, Shinohara $\mathrm{M}$ et al: Enhancement of active MMP release and invasive activity of lymph node metastatic tongue cancer cells by elevated signaling via the TNF-alpha-TNFR1-NF-kappaB pathway and a possible involvement of angiopoietin-like 4 in lung metastasis. Int J Onco/ 2016, 49(4):13771384.

56. Guo Y, Feng Y, Liu H, Luo S, Clarke JW, Moorman PG, Su L, Shen S, Christiani DC, Wei Q: Potentially functional genetic variants in the TNF/TNFR signaling pathway genes predict survival of patients with non-small cell lung cancer in the PLCO cancer screening trial. Mol Carcinog 2019, 58(7):10941104.

57. Zidi I, Mestiri S, Bartegi A, Amor NB: TNF-alpha and its inhibitors in cancer. Med Oncol 2010, 27(2):185-198.

58. Yuan X, Gajan A, Chu Q, Xiong H, Wu K, Wu GS: Developing TRAIL/TRAIL death receptor-based cancer therapies. Cancer Metastasis Rev 2018, 37(4):733-748.

59. Biswas S, Roy Chowdhury S, Mandal G, Purohit S, Gupta A, Bhattacharyya A: RelA driven coexpression of CXCL13 and CXCR5 is governed by a multifaceted transcriptional program regulating breast cancer progression. Biochim Biophys Acta Mol Basis Dis 2019, 1865(2):502-511.

60. Kochupurakkal BS, Wang ZC, Hua T, Culhane AC, Rodig SJ, Rajkovic-Molek K, Lazaro JB, Richardson AL, Biswas DK, Iglehart JD: RelA-Induced Interferon Response Negatively Regulates Proliferation. PLoS One 2015, 10(10):e0140243. 
61. Lesina M, Wormann SM, Morton J, Diakopoulos KN, Korneeva O, Wimmer M, Einwachter H, Sperveslage J, Demir IE, Kehl T et al: RelA regulates CXCL1/CXCR2-dependent oncogene-induced senescence in murine Kras-driven pancreatic carcinogenesis. J Clin Invest 2016, 126(8):2919-2932.

62. Ronin E, Lubrano di Ricco M, Vallion R, Divoux J, Kwon HK, Gregoire S, Collares D, Rouers A, Baud V, Benoist $C$ et al: The NF-kappaB RelA Transcription Factor Is Critical for Regulatory T Cell Activation and Stability. Front Immunol 2019, 10:2487.

63. Qu X, Han J, Zhang Y, Wang X, Fan H, Hua F, Yao R: TLR4-RelA-miR-30a signal pathway regulates Th17 differentiation during experimental autoimmune encephalomyelitis development. $J$ Neuroinflammation 2019, 16(1):183.

64. Concetti J, Wilson CL: NFKB1 and Cancer: Friend or Foe? Cells 2018, 7(9).

65. Li P, Gu J, Yang X, Cai H, Tao J, Yang X, Lu Q, Wang Z, Yin C, Gu M: Functional promoter -94 ins/del ATTG polymorphism in NFKB1 gene is associated with bladder cancer risk in a Chinese population. PLOS One 2013, 8(8):e71604.

66. Roskoski R, Jr.: Src protein-tyrosine kinase structure and regulation. Biochem Biophys Res Commun 2004, 324(4):1155-1164.

67. Venkadakrishnan VB, DePriest AD, Kumari S, Senapati D, Ben-Salem S, Su Y, Mudduluru G, Hu Q, Cortes E, Pop E et al: Protein Kinase N1 control of androgen-responsive serum response factor action provides rationale for novel prostate cancer treatment strategy. Oncogene 2019, 38(23):4496-4511.

68. Franko A, Kovarova M, Feil S, Feil R, Wagner R, Heni M, Konigsrainer A, Ruoss M, Nussler AK, Weigert $C$ et al: cGMP-dependent protein kinase I (cGKI) modulates human hepatic stellate cell activation. Metabolism 2018, 88:22-30.

69. Schuler F, Afreen S, Manzl C, Hacker G, Erlacher M, Villunger A: Checkpoint kinase 1 is essential for fetal and adult hematopoiesis. EMBO Rep 2019, 20(8):e47026.

70. Browning DD, Kwon IK, Wang R: cGMP-dependent protein kinases as potential targets for colon cancer prevention and treatment. Future Med Chem 2010, 2(1):65-80.

71. Lin W, Chen S: Checkpoint Kinase 1 Is Overexpressed during HPV16-Induced Cervical Carcinogenesis. Gynecol Obstet Invest 2018, 83(3):299-305.

72. Lamar JM, Xiao Y, Norton E, Jiang ZG, Gerhard GM, Kooner S, Warren JSA, Hynes RO: SRC tyrosine kinase activates the YAP/TAZ axis and thereby drives tumor growth and metastasis. $J$ Biol Chem 2019, 294(7):2302-2317.

73. Mao L, Liu S, Hu L, Jia L, Wang H, Guo M, Chen C, Liu Y, Xu L: miR-30 Family: A Promising Regulator in Development and Disease. Biomed Res Int 2018, 2018:9623412.

74. Yin H, Wang Y, Wu Y, Zhang X, Zhang X, Liu J, Wang T, Fan J, Sun J, Yang A et al: EZH2-mediated Epigenetic Silencing of miR-29/miR-30 targets LOXL4 and contributes to Tumorigenesis, Metastasis, and Immune Microenvironment Remodeling in Breast Cancer. Theranostics 2020, 10(19):8494-8512.

75. Jiang S, Miao D, Wang M, Lv J, Wang Y, Tong J: MiR-30-5p suppresses cell chemoresistance and stemness in colorectal cancer through USP22/Wnt/beta-catenin signaling axis. J Cell Mol Med 2019, 23(1):630-640. 
76. Farhood B, Najafi M, Mortezaee K: CD8(+) cytotoxic T lymphocytes in cancer immunotherapy: A review. J Cell Physiol 2019, 234(6):8509-8521.

77. Bremnes RM, Busund LT, Kilvaer TL, Andersen S, Richardsen E, Paulsen EE, Hald S, Khanehkenari MR, Cooper WA, Kao SC et al: The Role of Tumor-Infiltrating Lymphocytes in Development, Progression, and Prognosis of Non-Small Cell Lung Cancer. J Thorac Oncol 2016, 11(6):789-800.

\section{Tables}

Table 1ロThe mRNA levels of CXC chemokines with differential expression in BLCA tissues and normal bladder mucosa tissues (ONCOMINE).

\begin{tabular}{llcccc}
\hline TLR & Type & Fold change & P-value & t-test & References \\
\hline CXCL2 & Superficial Bladder Cancer & -12.716 & $7.74 \mathrm{E}-17$ & -11.618 & $(38)$ \\
CXCL3 & Superficial Bladder Cancer & -3.564 & $2.44 \mathrm{E}-08$ & -6.122 & $(38)$ \\
\hline CXCL6 & Infiltrating Bladder Urothelial Carcinoma & 2.272 & $1.34 \mathrm{E}-06$ & 5.02 & $(38)$ \\
\hline CXCL9 & Infiltrating Bladder Urothelial Carcinoma & 2.84 & $3.92 \mathrm{E}-09$ & 6.197 & $(38)$ \\
\hline CXCL10 & Infiltrating Bladder Urothelial Carcinoma & 3.039 & $6.79 \mathrm{E}-11$ & 7.04 & $(38)$ \\
\hline CXCL12 & Superficial Bladder Cancer & -4.495 & $2.52 \mathrm{E}-21$ & -12.211 & $(39)$ \\
\hline & Infiltrating Bladder Urothelial Carcinoma & -3.371 & $1.41 \mathrm{E}-12$ & -7.727 & $(39)$ \\
\hline & Superficial Bladder Cancer & -2.531 & $1.92 \mathrm{E}-12$ & -8.846 & $(38)$ \\
\hline CXCL13 & Infiltrating Bladder Urothelial Carcinoma & 6.369 & $1.07 \mathrm{E}-10$ & 7.171 & $(38)$ \\
\hline
\end{tabular}

Table 2ロKey regulated factor of CXC chemokines in BLCA (TRRUST).

\begin{tabular}{|c|c|c|c|c|c|}
\hline Key TF & Description & $\begin{array}{l}\text { Overlapped } \\
\text { genes }\end{array}$ & $\begin{array}{c}\mathrm{P} \\
\text { value }\end{array}$ & $\begin{array}{c}\mathrm{Q} \\
\text { value }\end{array}$ & List of overlapped genes \\
\hline RELA & $\begin{array}{l}\text { v-rel reticuloendotheliosis viral oncogene } \\
\text { homolog A (avian) }\end{array}$ & 5 & $\begin{array}{l}1.16 \mathrm{E}- \\
06\end{array}$ & $\begin{array}{l}1.79 \mathrm{E}- \\
06\end{array}$ & $\begin{array}{c}\text { CXCL10 } \\
\text {,CXCL12,CXCL2,CXCL5,CXCL1 }\end{array}$ \\
\hline NFKB1 & $\begin{array}{l}\text { nuclear factor of kappa light polypeptide } \\
\text { gene enhancer in B-cells } 1\end{array}$ & 6 & $\begin{array}{l}1.16 \mathrm{E}- \\
06\end{array}$ & $\begin{array}{l}1.79 \mathrm{E}- \\
06\end{array}$ & CXCL12,CXCL10,CXCL5,CXCL1,CXCL2 \\
\hline SP1 & Sp1 transcription factor & 2 & 0.0406 & 0.0406 & CXCL5,CXCL1 \\
\hline
\end{tabular}

Table 3: The Kinase target networks of CXC chemokines in BLCA (LinkedOmics). 


\begin{tabular}{|c|c|c|c|c|}
\hline $\begin{array}{c}\text { CXC } \\
\text { chemokines }\end{array}$ & Kinase target & Description & Leading Edge Number & $\begin{array}{l}\mathrm{P} \\
\text { Value }\end{array}$ \\
\hline CXCL1 & Kinase_LYN & LYN proto-oncogene, Src family tyrosine kinase & 17 & 0 \\
\hline \multirow{3}{*}{ CXCL2 } & Kinase_IKBKB & $\begin{array}{l}\text { inhibitor of nuclear factor kappa B kinase subunit } \\
\text { beta }\end{array}$ & 9 & 0 \\
\hline & Kinase_LCK & LCK proto-oncogene, Src family tyrosine kinase & 24 & 0 \\
\hline & Kinase_LYN & LYN proto-oncogene, Src family tyrosine kinase & 21 & 0 \\
\hline \multirow[t]{2}{*}{ CXCL3 } & Kinase_LYN & LYN proto-oncogene, Src family tyrosine kinase & 19 & 0 \\
\hline & Kinase_LCK & LCK proto-oncogene, Src family tyrosine kinase & 25 & 0 \\
\hline \multirow[t]{2}{*}{ CXCL5 } & Kinase_LCK & LCK proto-oncogene, Src family tyrosine kinase & 24 & 0 \\
\hline & Kinase_LYN & LYN proto-oncogene, Src family tyrosine kinase & 18 & 0 \\
\hline \multirow[t]{2}{*}{ CXCL6 } & Kinase_SYK & spleen associated tyrosine kinase & 16 & 0 \\
\hline & Kinase_LYN & LYN proto-oncogene, Src family tyrosine kinase & 24 & 0 \\
\hline \multirow[t]{2}{*}{ CXCL7 } & Kinase_PKN1 & protein kinase N1 & 5 & 0.009 \\
\hline & Kinase_ADRBK1 & G protein-coupled receptor kinase 2 & 10 & 0 \\
\hline \multirow[t]{2}{*}{ CXCL9 } & Kinase_LYN & LYN proto-oncogene, Src family tyrosine kinase & 18 & 0 \\
\hline & Kinase_LCK & LCK proto-oncogene, Src family tyrosine kinase & 20 & 0 \\
\hline \multirow[t]{2}{*}{ CXCL10 } & Kinase_LCK & LCK proto-oncogene, Src family tyrosine kinase & 20 & 0 \\
\hline & Kinase_LYN & LYN proto-oncogene, Src family tyrosine kinase & 16 & 0 \\
\hline \multirow[t]{2}{*}{ CXCL11 } & Kinase_LCK & LCK proto-oncogene, Src family tyrosine kinase & 20 & 0 \\
\hline & Kinase_LYN & LYN proto-oncogene, Src family tyrosine kinase & 17 & 0 \\
\hline \multirow[t]{2}{*}{ CXCL12 } & Kinase_LYN & LYN proto-oncogene, Src family tyrosine kinase & 20 & 0 \\
\hline & Kinase_PRKG1 & protein kinase, cGMP-dependent, type I & 15 & 0.004 \\
\hline \multirow[t]{2}{*}{ CXCL13 } & Kinase_LCK & LCK proto-oncogene, Src family tyrosine kinase & 22 & 0 \\
\hline & Kinase_LYN & LYN proto-oncogene, Src family tyrosine kinase & 19 & 0 \\
\hline \multirow[t]{2}{*}{ CXCL16 } & Kinase_LCK & LCK proto-oncogene, Src family tyrosine kinase & 22 & 0 \\
\hline & Kinase_JAK2 & Janus kinase 2 & 7 & 0 \\
\hline \multirow[t]{2}{*}{ CXCL17 } & Kinase_CHEK1 & checkpoint kinase 1 & 45 & 0 \\
\hline & Kinase_PLK1 & polo like kinase 1 & 41 & 0 \\
\hline
\end{tabular}

Table 4: The miRNA target networks of CXC chemokines in BLCA (LinkedOmics).

\begin{tabular}{llrr}
\hline CXC chemokines & MIRNA targets & Leading Edge Number & P Value \\
\hline CXCL1 & GTAAGAT,MIR-200A & 20 & 133 \\
& TGTTTAC,MIR-30A-5P,MIR-30C,MIR-30D,MIR-30B,MIR-30E-5P & 47 \\
CXCL3 & AACATTC,MIR-409-3P & 24 & 0.009 \\
\hline CXCL5 & CTCAAGA,MIR-526B & 72 & 0.009 \\
\cline { 2 - 4 } & CAGTGTT,MIR-141,MIR-200A & 89 & 0.019 \\
\hline CXCL6 & TATTATA,MIR-374 & 192 & 0.015 \\
\cline { 2 - 4 } & TGTTTAC,MIR-30A-5P,MIR-30C,MIR-30D,MIR-30B,MIR-30E-5P & 21 \\
\hline CXCL7 & GTATGAT,MIR-154,MIR-487 & 7 & 0 \\
\hline CXCL17 & GCGCCTT,MIR-525,MIR-524 & 0 & 10 \\
\hline
\end{tabular}

Table 5: The cox proportional hazard model of CXC chemokines and six tumor-infiltrating immune cells in BLCA (TIMER2.0). 


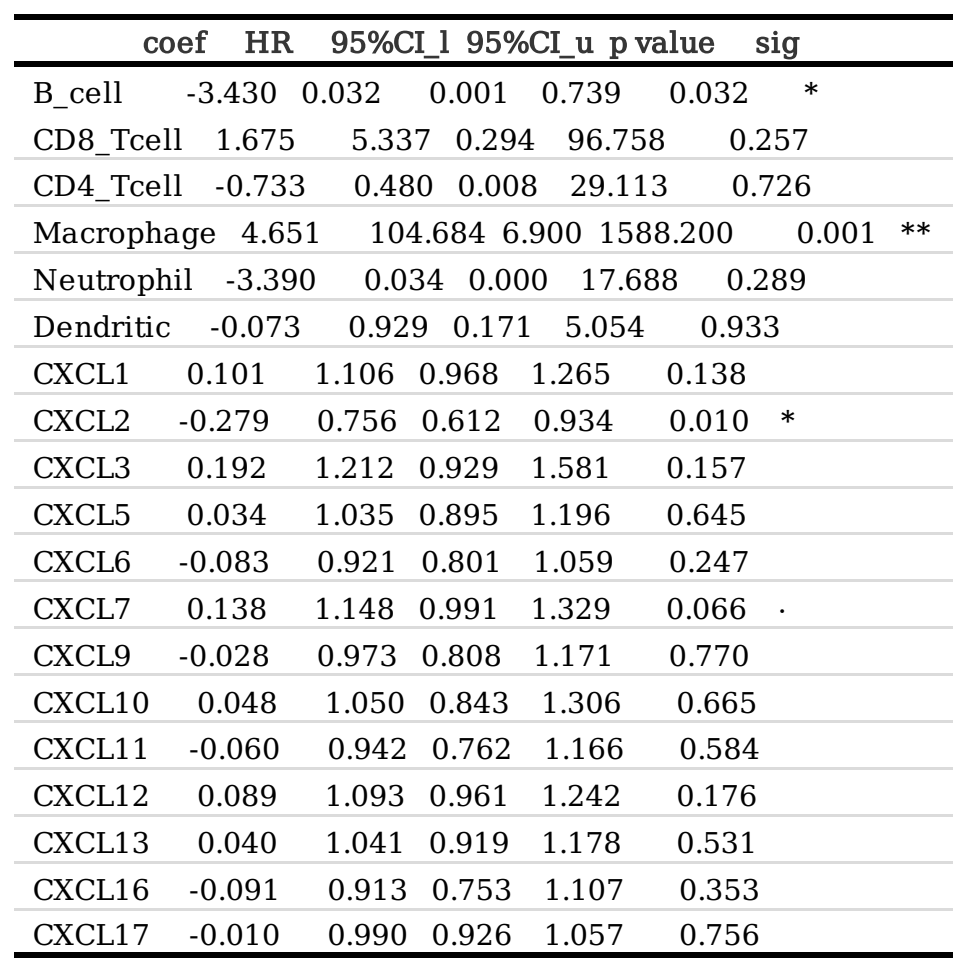

Figures

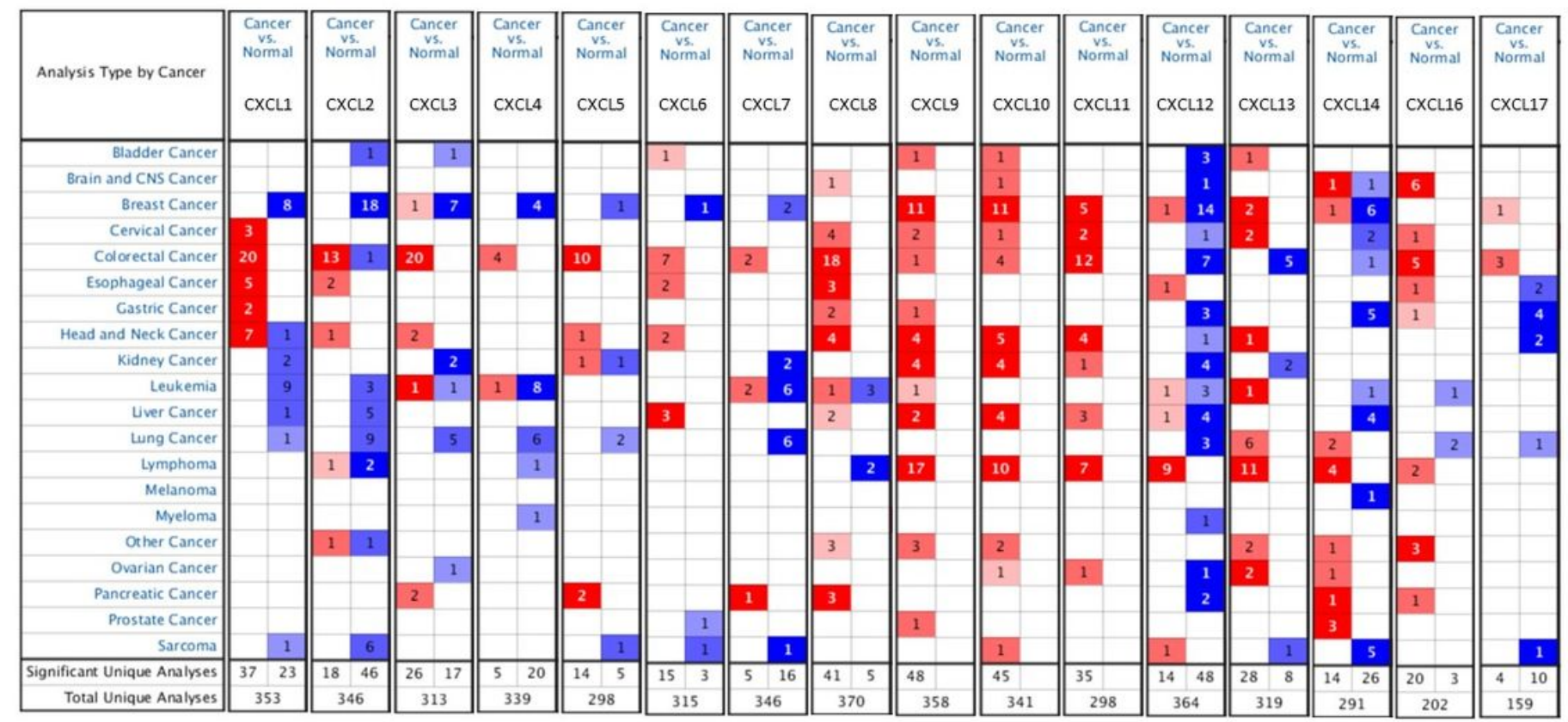

붐ำ

Figure 1 
CXC chemokine mRNA levels in bladder cancer (Oncomine). Red and blue represent significantly overexpressed and downregulate mRNAs, respectively.
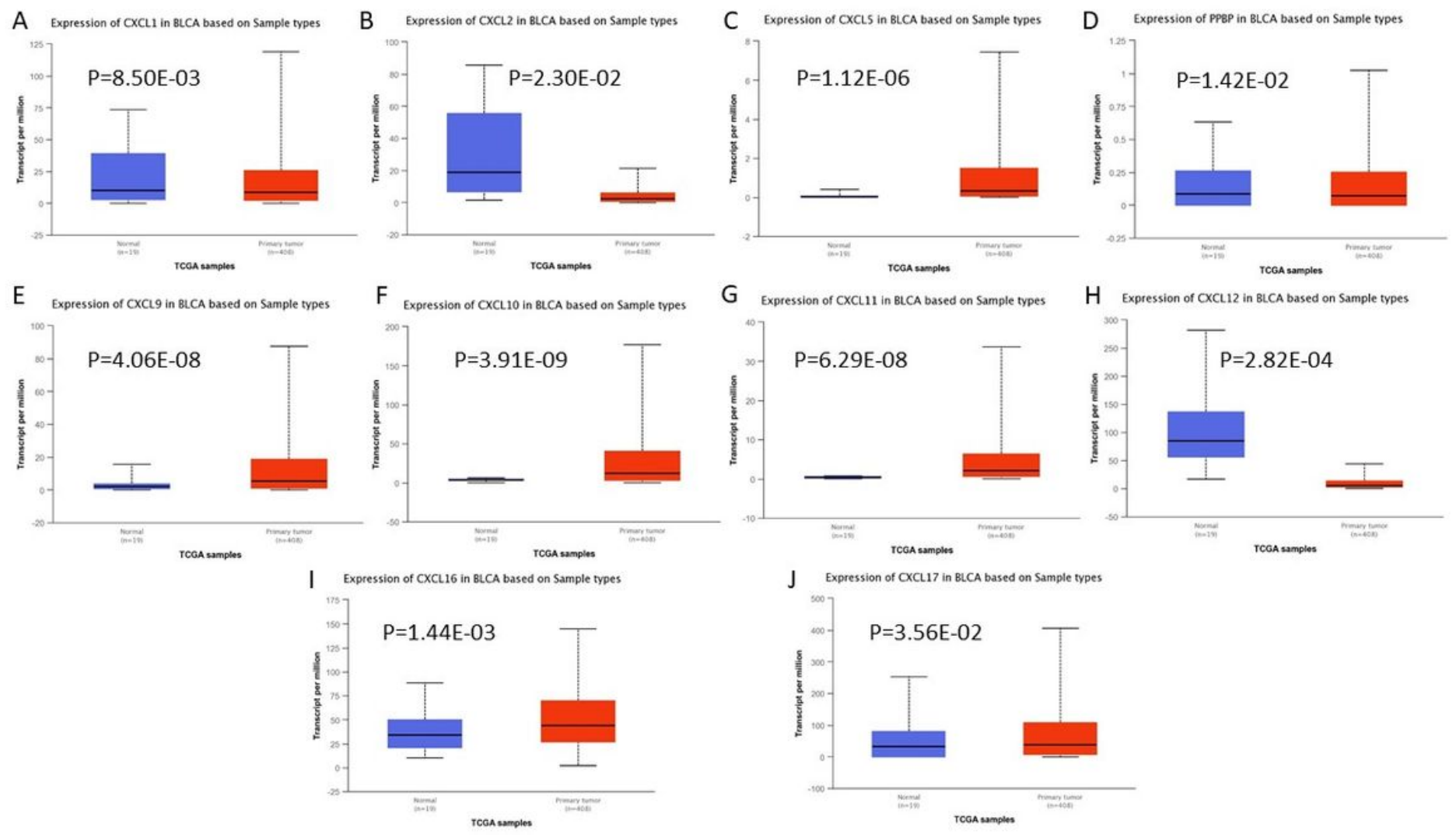

H Expression of CXCL12 in BLCA based on Sample types

Figure 2

CXC chemokine mRNA levels in bladder cancer (UALCAN). The mRNA levels of (A) CXCL1, (C) CXCL5, (D) CXCL7, (E) CXCL9, (F) CXCL10, (G) CXCL11, (I) CXCL16, and (J) CXCL17 were significantly elevated and the mRNA levels of (B) CXCL2, $(H)$ CXCL12 were significantly reduced bladder cancer tissues. CXCL, C-X$\mathrm{C}$ motif chemokine ligand. 


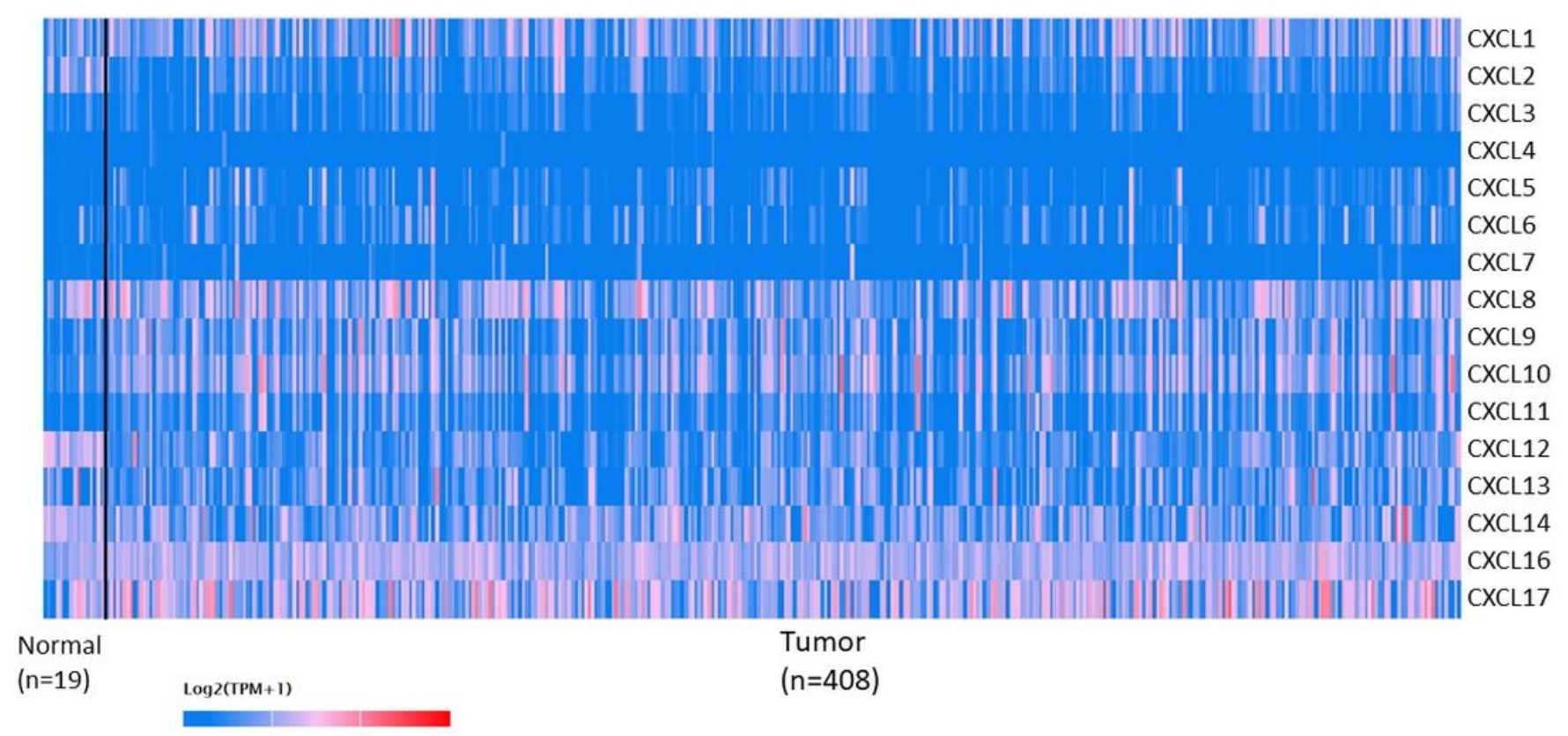

Figure 3

CXC chemokine relative protein levels in bladder cancer $(n=408)$ and healthy tissues $(n=19)$ (UALCAN).
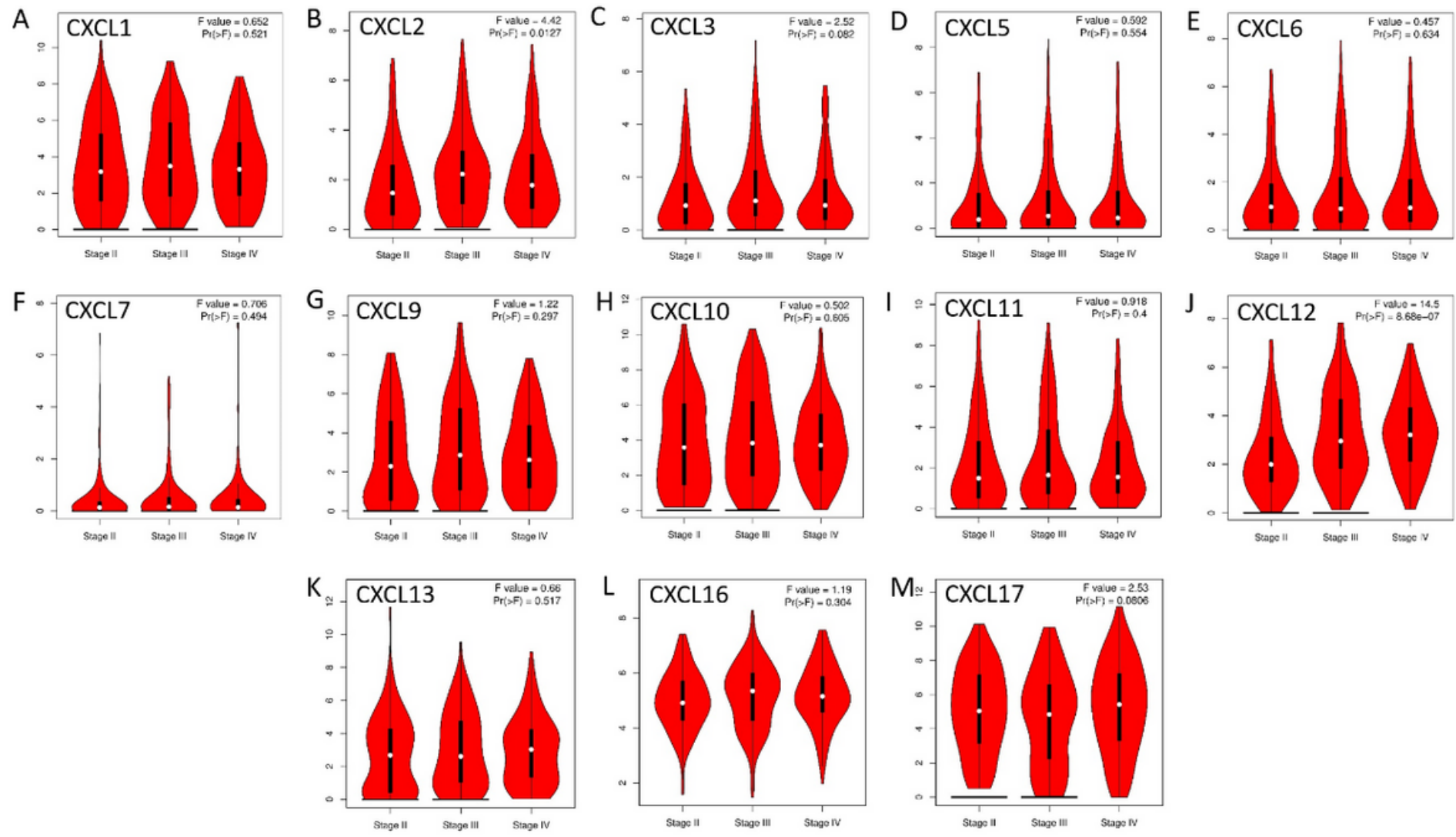
Figure 4

Correlation between the pathological stage of patients with bladder cancer and differentially expressed CXC chemokines (GEPIA). (A) CXCL1, (B) CXCL2, (C) CXCL3, (D) CXCL5, (E) CXCL6, (F) CXCL7, (G) CXCL9, (H) CXCL10, (I) CXCL11, (J) CXCL12, (K) CXCL13, (L) CXCL16, and (M) CXCL17. CXCL, C-X-C motif chemokine ligand.
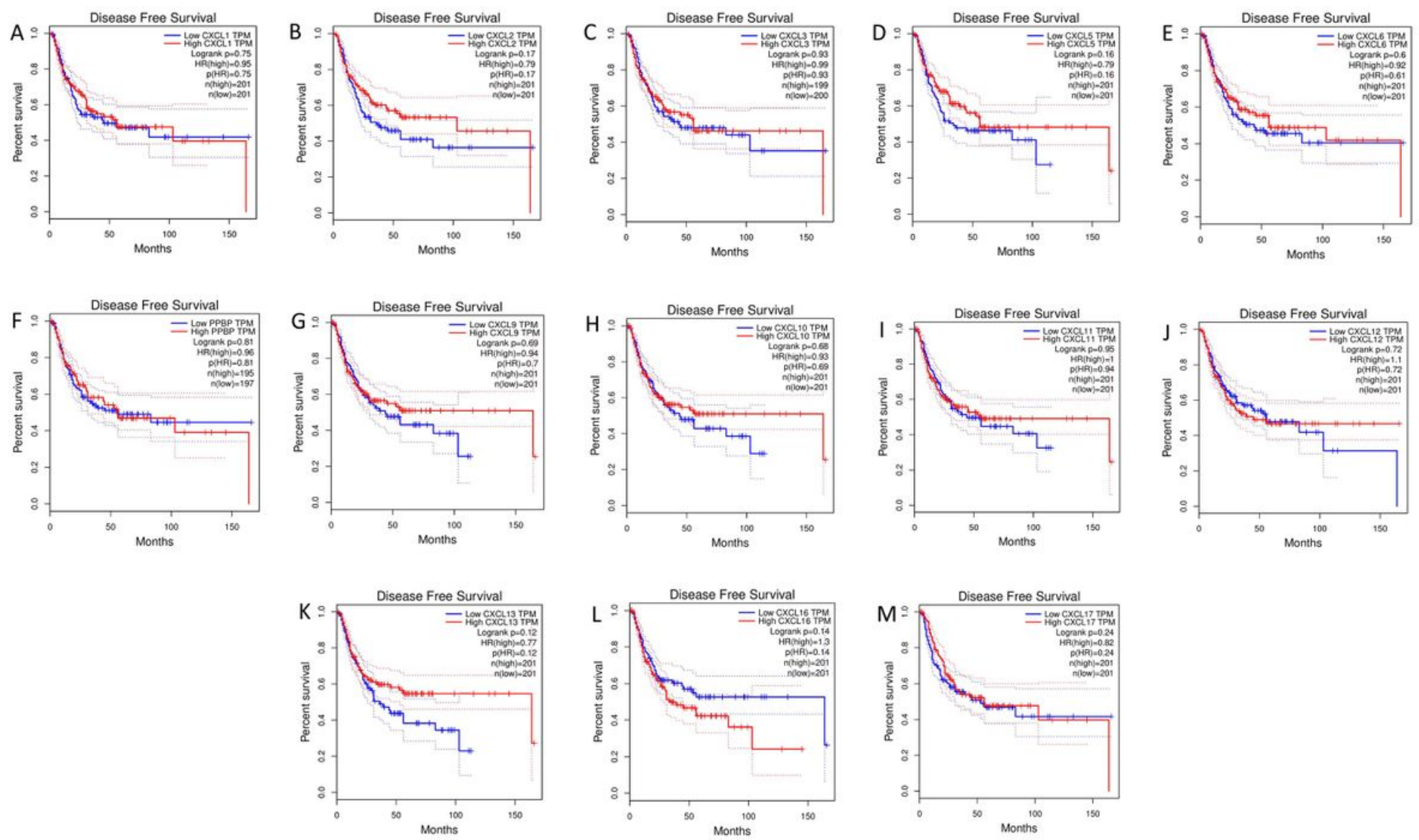

\section{Figure 5}

The ability of differentially expressed CXC chemokines to predict the prognosis (disease free survival) of patients with bladder cancer (GEPIA). (A) CXCL1, (B) CXCL2, (C) CXCL3, (D) CXCL5, (E) CXCL6, (F) CXCL7, (G) CXCL9, (H) CXCL10, (I) CXCL11, (J) CXCL12, (K) CXCL13, (L) CXCL16, and (M) CXCL17. CXCL, C-X-C motif chemokine ligand. 

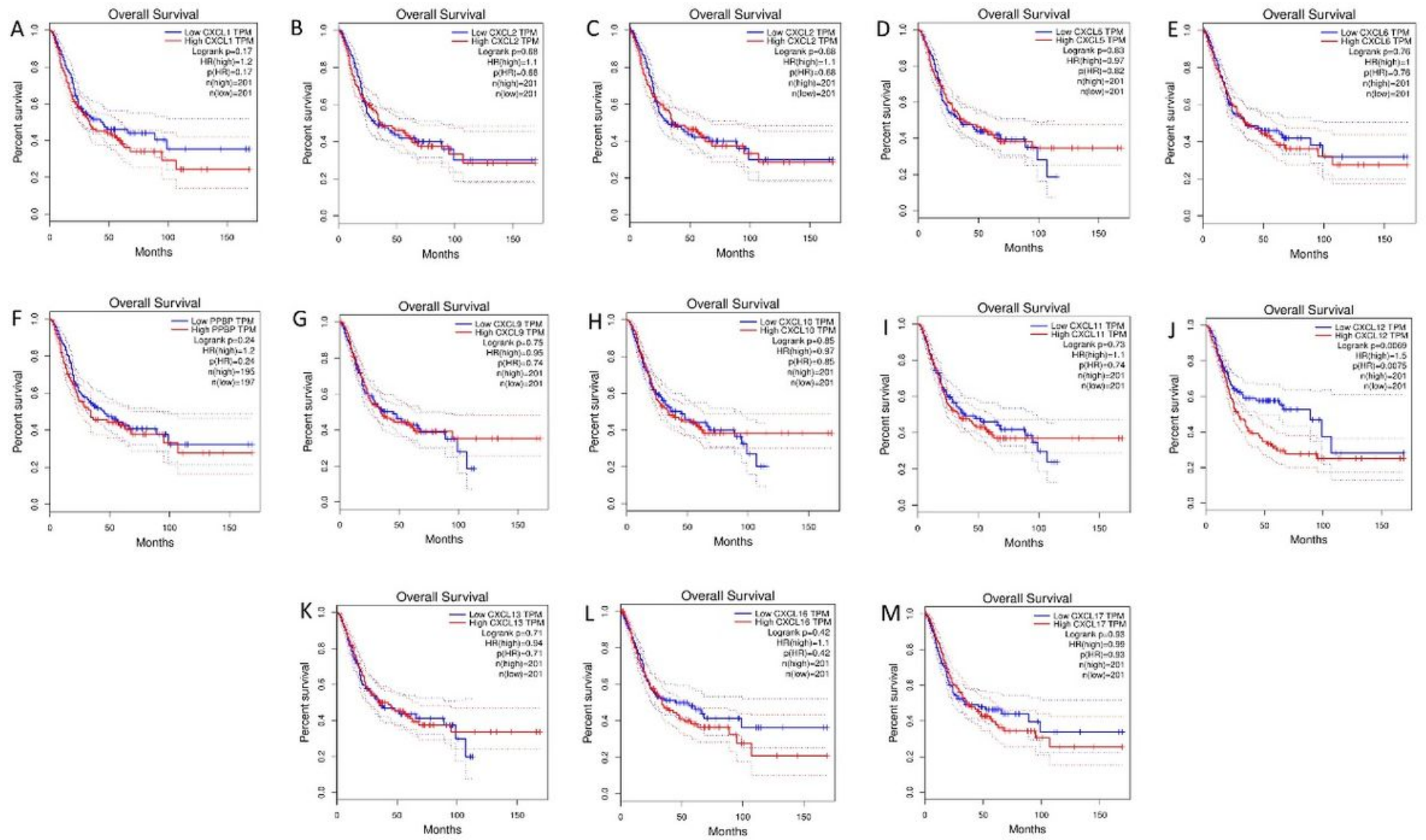

\section{Figure 6}

The ability of differentially expressed $C X C$ chemokines to predict the prognosis (overall survival) of patients with bladder cancer (GEPIA). (A) CXCL1, (B) CXCL2, (C) CXCL3, (D) CXCL5, (E) CXCL6, (F) CXCL7, (G) CXCL9, (H) CXCL10, (I) CXCL11, (J) CXCL12, (K) CXCL13, (L) CXCL16, and (M) CXCL17. CXCL, C-X-C motif chemokine ligand. 

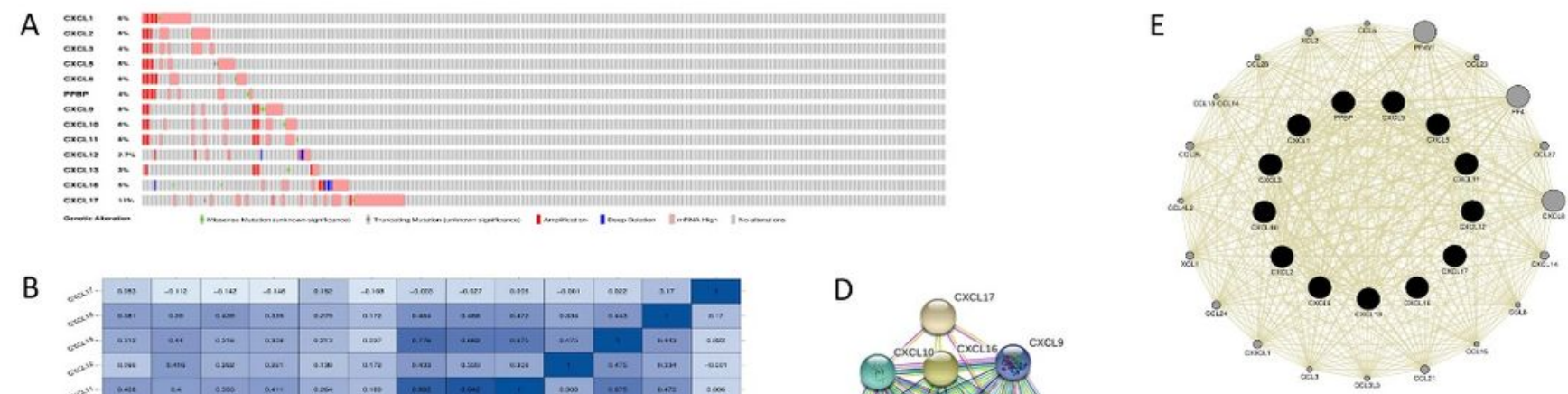

B
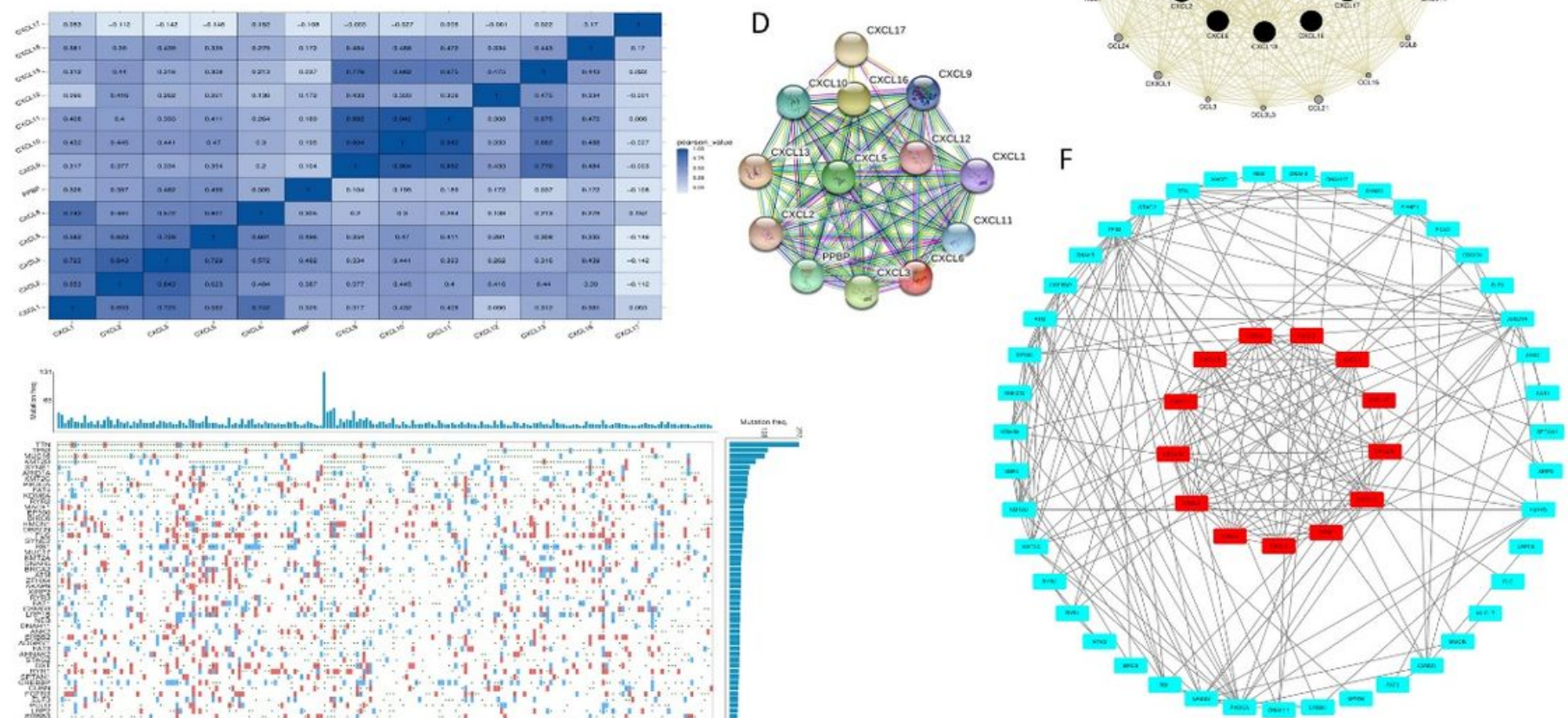

Figure 7

Interaction analyses between genetic alteration, the 50 top mutated genes, and differentially expressed CXC chemokines in patients with bladder cancer. (A) genetic alterations of differentially expressed CXC chemokines. (B) Heatmap of the correlations of differentially expressed CXC chemokines. (C) The 50 top mutated genes and their mutation frequencies. (D, E) Differentially expressed CXC chemokines' proteinprotein interaction network. (F) Gene-gene interaction network of the 50 top mutated genes and the differentially expressed CXC chemokines. 
A
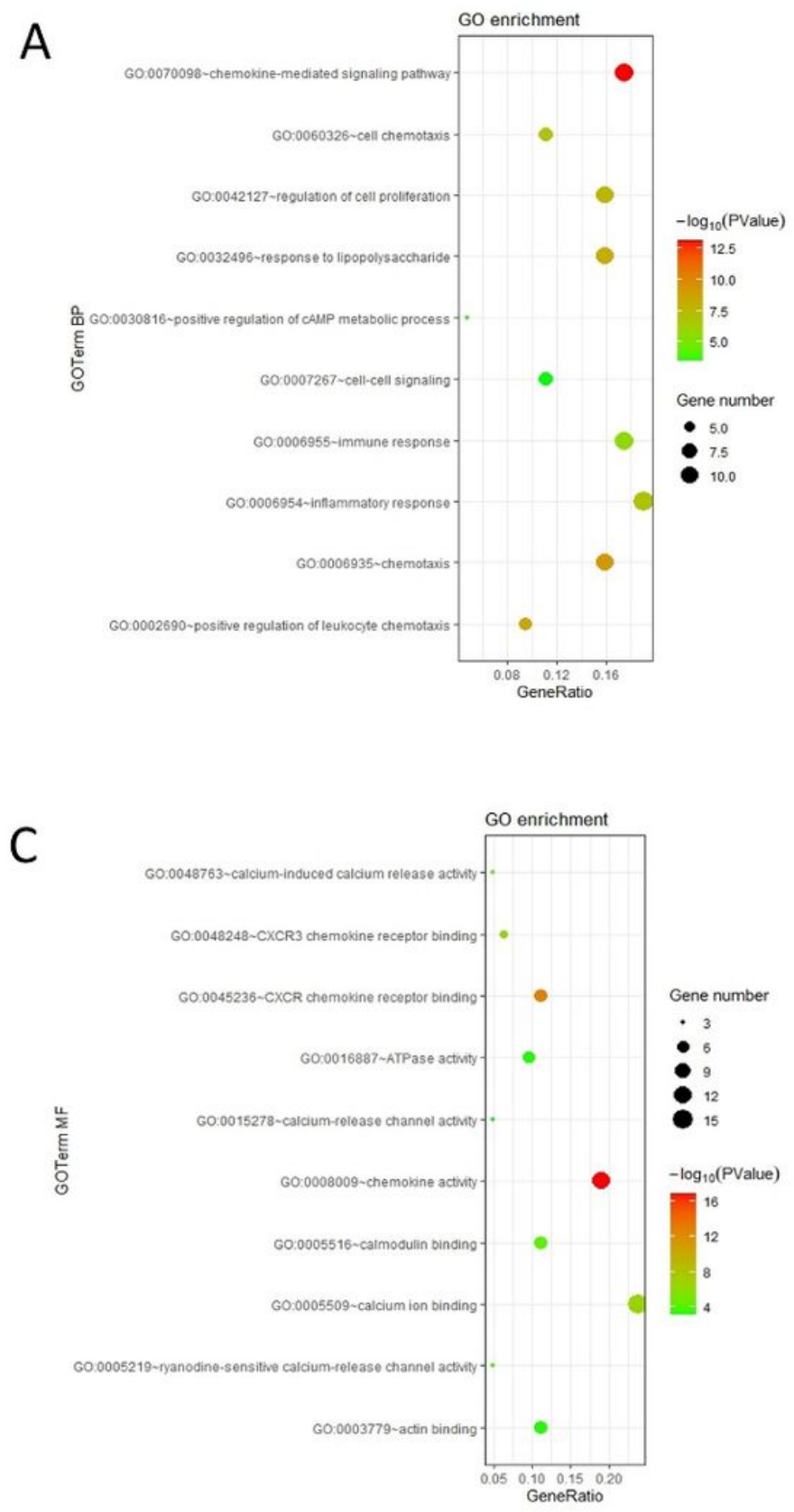

B

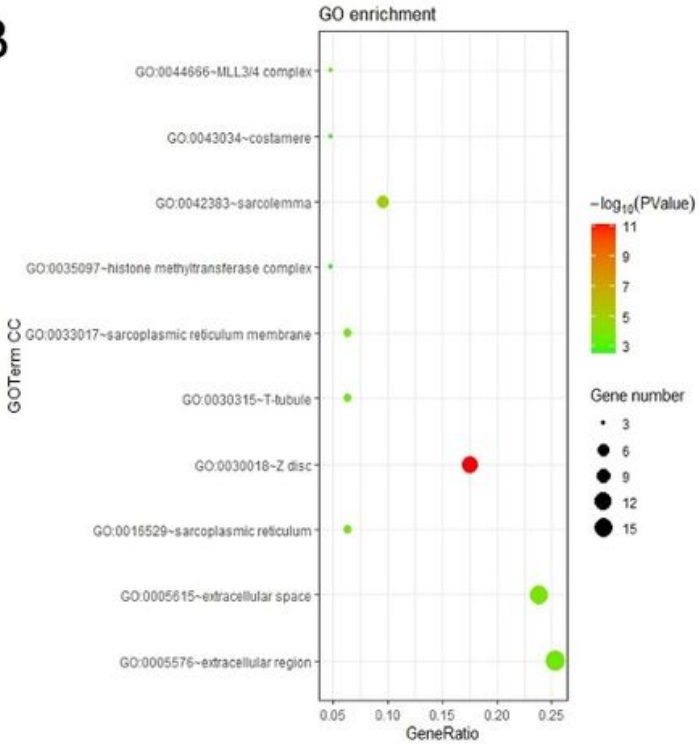

D

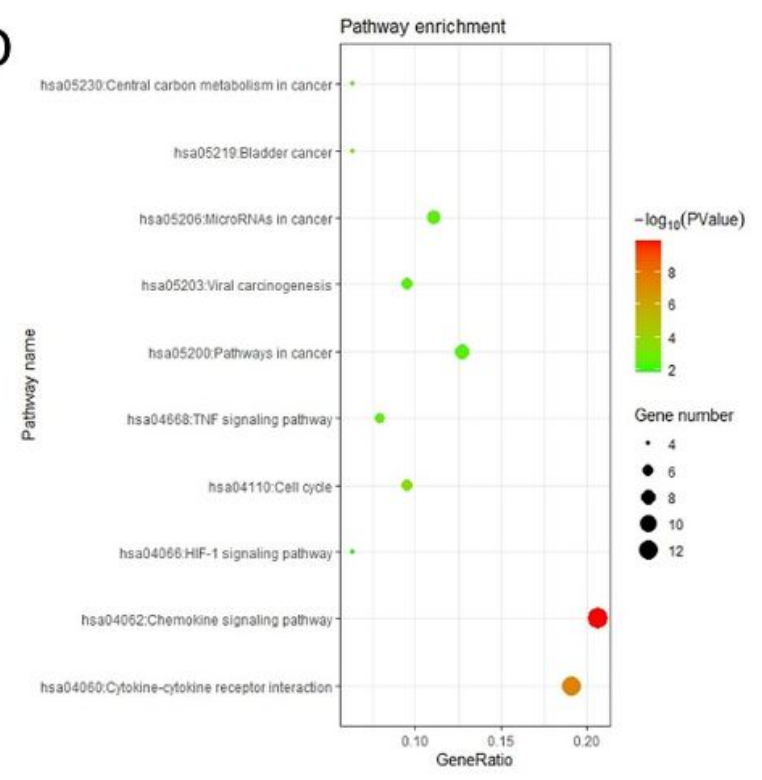

\section{Figure 8}

Enrichment analysis of the 50 top mutated genes and differentially expressed CXC chemokines in bladder cancer (David 6.8). Bubble plot of enriched $G 0$ terms for biological process (A), cellular component (B) and molecular function (C) categories. Bubble plot of enriched KEGG terms (D). Go, gene ontology; KEGG, Kyoto Encyclopedia of Genes and Genomes. 
A

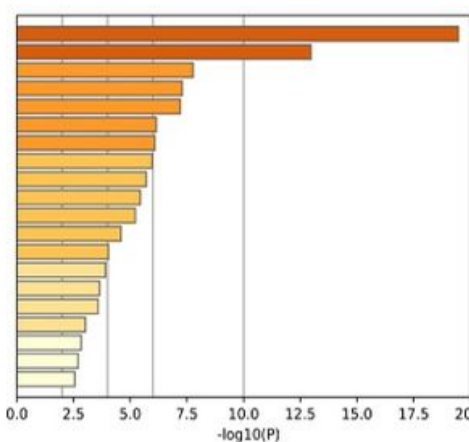

B

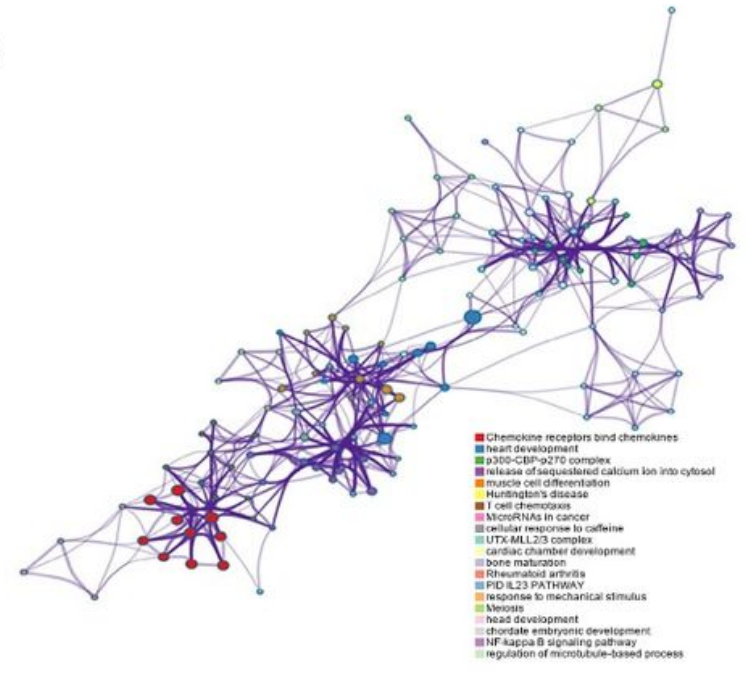

C
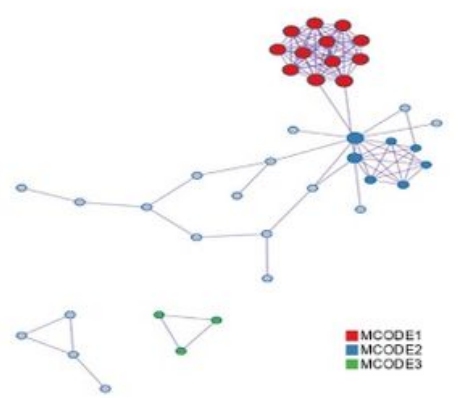

MCODE1
MCODE2
MCODE
MCOOE

D
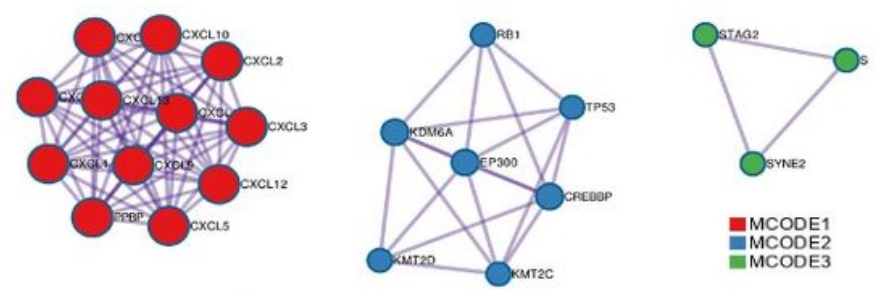

EMCODE1 - MCODE2

E

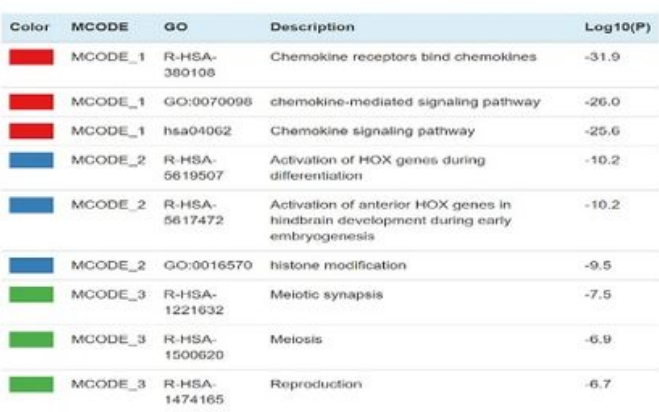

Figure 9

Enrichment analysis of the 50 top mutated genes and differentially expressed CXC chemokines in bladder cancer (Metascape). (A) Bar plot show in the top 20 terms enriched for the 50 top mutated genes and differentially expressed CXC chemokines. (B) The network of enriched terms. (C, D, E) MCODE components and protein-protein interaction network identified for the 50 top mutated genes and differentially expressed CXC chemokines. 


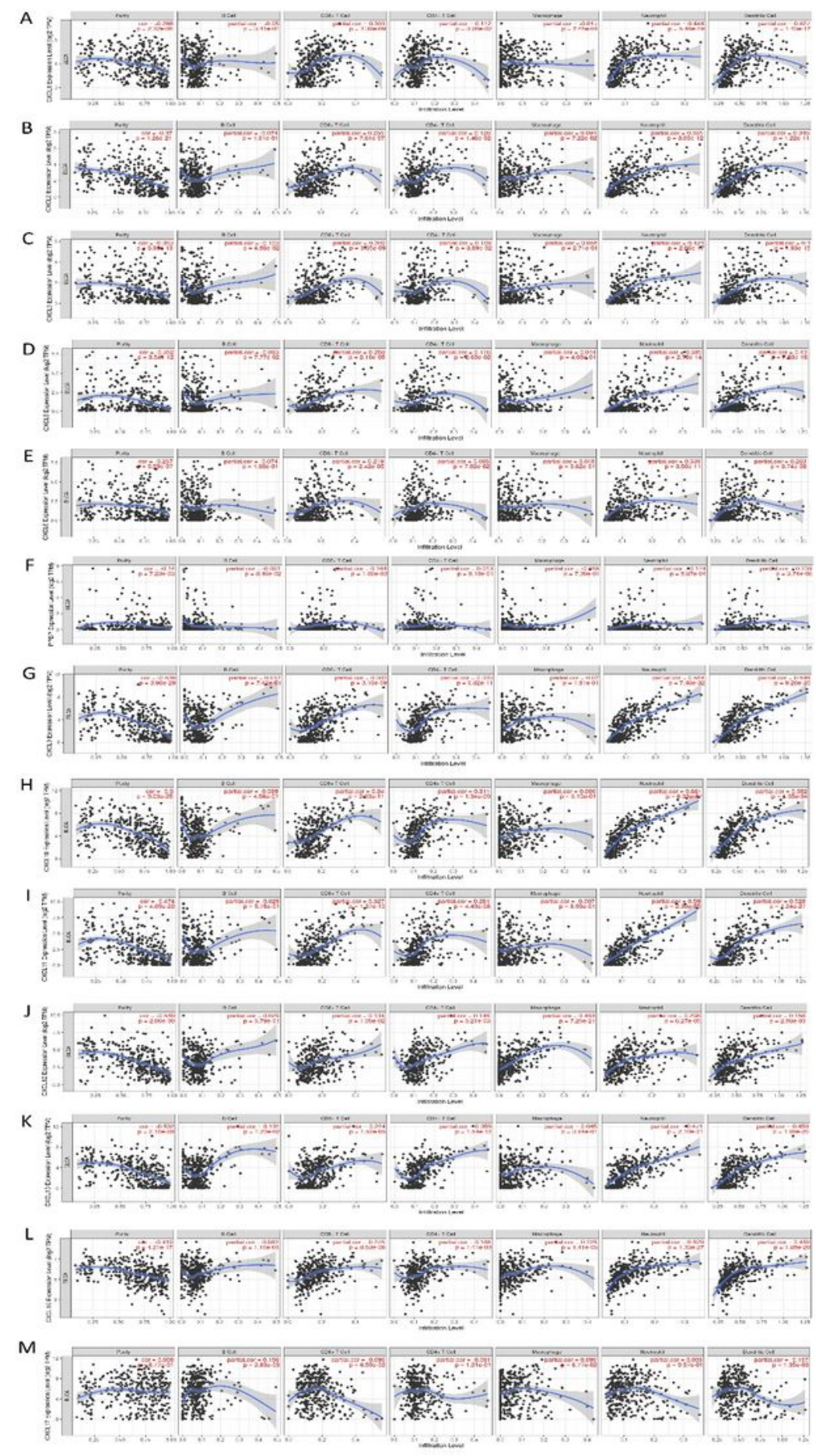

\section{Figure 10}

Correlation between immune cell infiltration and differentially expressed CXC chemokines (TIMER2.0). (A) CXCL1, (B) CXCL2, (C) CXCL3, (D) CXCL5, (E) CXCL6, (F) CXCL7, (G) CXCL9, (H) CXCL10, (I) CXCL11, (J) CXCL12, (K) CXCL13, (L) CXCL16, and (M) CXCL17 in bladder cancer. CXCL, C-X-C motif chemokine ligand. 

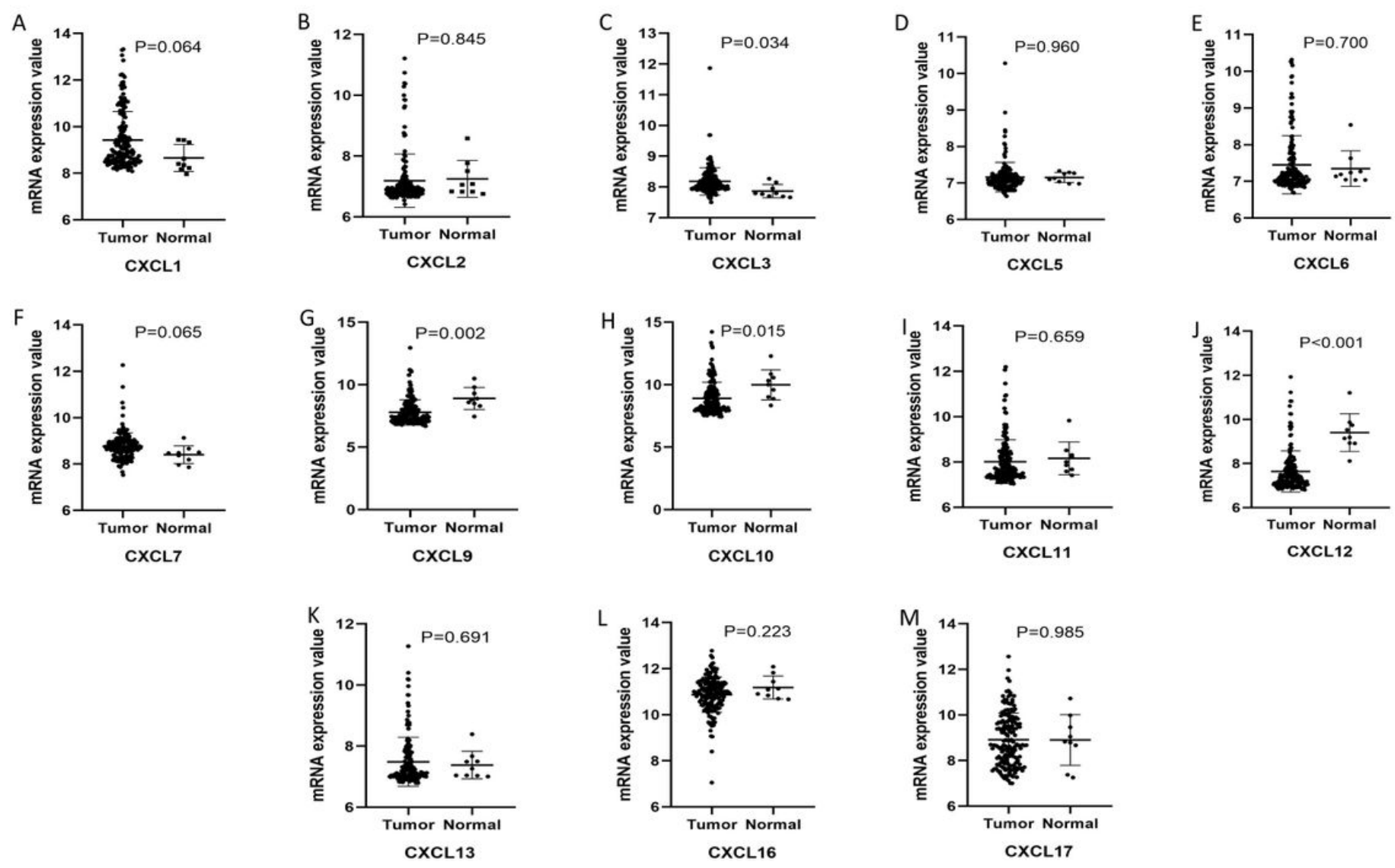

Figure 11

CXC chemokine mRNA levels in bladder cancer and normal tissues (GE013507). (A) CXCL1, (B) CXCL2, (C) CXCL3, (D) CXCL5, (E) CXCL6, (F) CXCL7, (G) CXCL9, (H) CXCL10, (I) CXCL11, (J) CXCL12, (K) CXCL13, (L) CXCL16, and (M) CXCL17. CXCL, C-X-C motif chemokine ligand. 
A

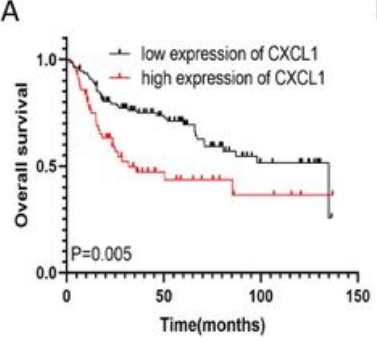

B
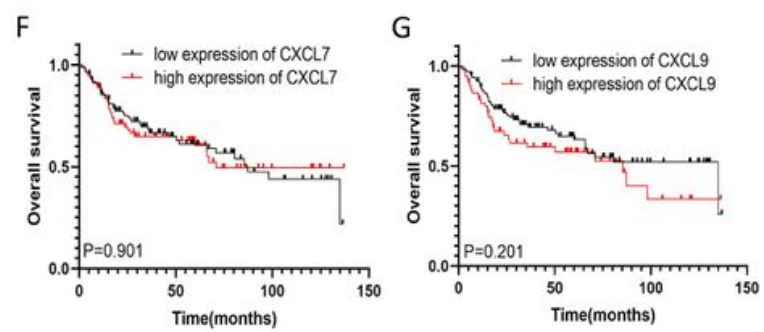

K
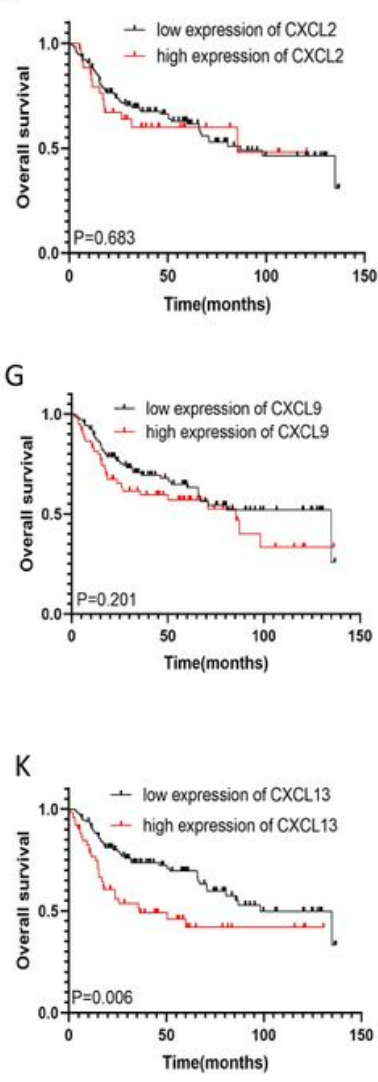

G
C

$\mathrm{H}$
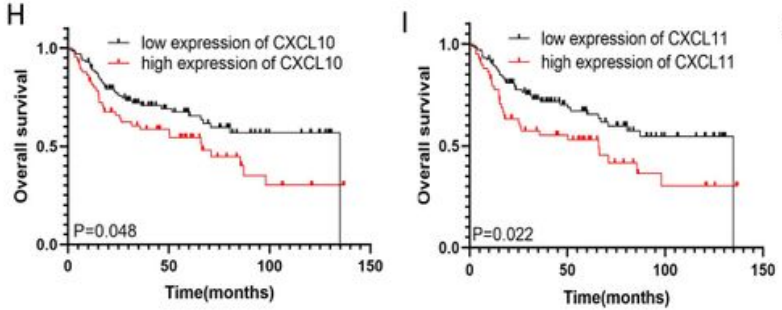

L

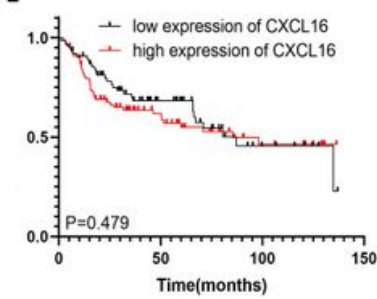

D
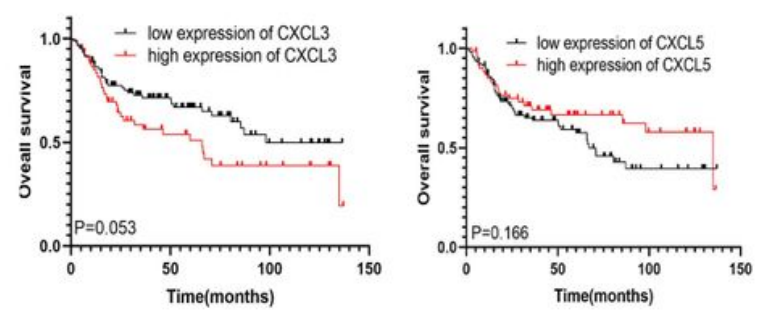

M
E
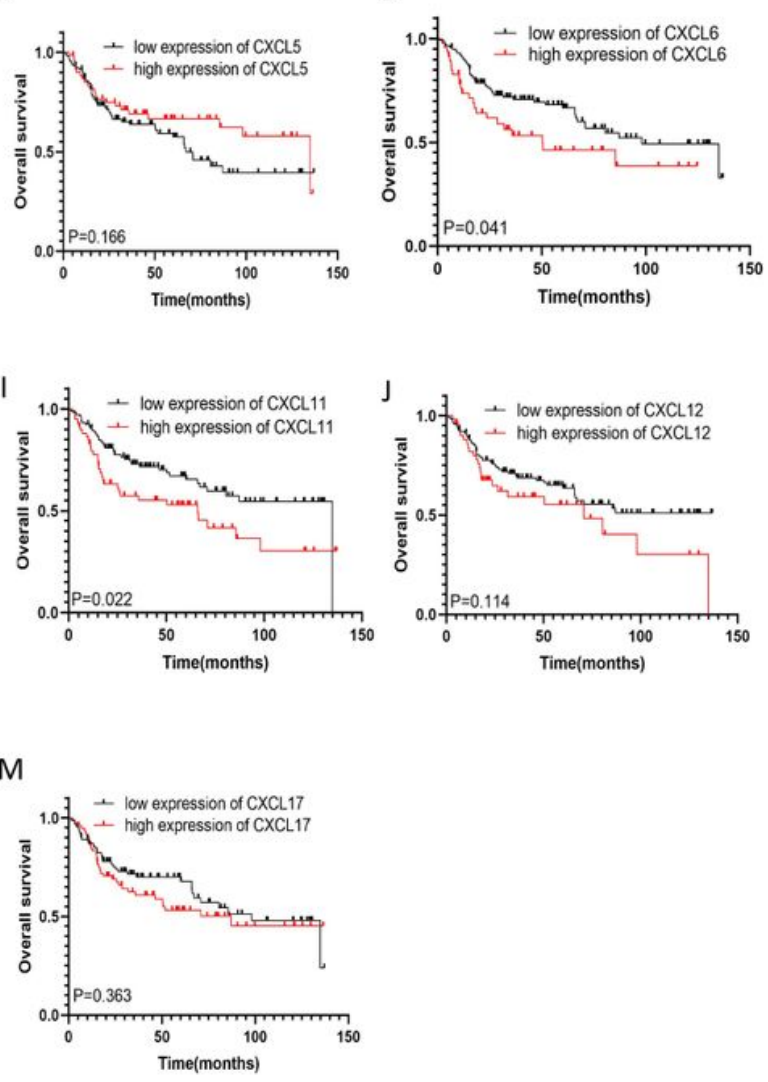

Figure 12

The ability of differentially expressed CXC chemokines to predict the prognosis (overall survival) of patients with bladder cancer (GE013507). (A) CXCL1, (B) CXCL2, (C) CXCL3, (D) CXCL5, (E) CXCL6, (F) CXCL7, (G) CXCL9, (H) CXCL10, (I) CXCL11, (J) CXCL12, (K) CXCL13, (L) CXCL16, and (M) CXCL17. CXCL, $\mathrm{C}-\mathrm{X}-\mathrm{C}$ motif chemokine ligand. 